TRANSACTIONS OF THE

AMERICAN MATHEMATICAL SOCIETY

Volume 363, Number 4, April 2011, Pages 2003-2028

S 0002-9947(2010)05051-2

Article electronically published on November 5, 2010

\title{
CONTINUITY IN WEAK TOPOLOGY AND EXTREMAL PROBLEMS OF EIGENVALUES OF THE $p$-LAPLACIAN
}

\author{
PING YAN AND MEIRONG ZHANG
}

\begin{abstract}
We will study the dependence of eigenvalues of the one-dimensional $p$-Laplacian on potentials or weights. Two results are obtained. One is the continuity of eigenvalues in potentials with respect to the weak topologies of $L^{\gamma}$ spaces, $1 \leq \gamma \leq \infty$, and the other is the continuous differentiability of eigenvalues in potentials with respect to $L^{\gamma}$ norms. As applications, we will study some extremal problems of eigenvalues by developing some analytical methods.
\end{abstract}

\section{INTRODUCTION}

Given an exponent $1<p<\infty$, define the mapping $\phi_{p}: \mathbb{R} \rightarrow \mathbb{R}$ by

$$
\phi_{p}(x):=|x|^{p-2} x .
$$

For a potential $q \in \mathcal{L}^{\gamma}:=L^{\gamma}([0,1], \mathbb{R}), \gamma \in[1, \infty]$, we consider the eigenvalue problem of the one-dimensional $p$-Laplacian

$$
\left(\phi_{p}\left(x^{\prime}\right)\right)^{\prime}+(\lambda+q(t)) \phi_{p}(x)=0 \quad\left({ }^{\prime}=\frac{d}{d t}\right)
$$

with the separated boundary condition

$$
c_{11} x(0)+c_{12} x^{\prime}(0)=0, \quad c_{21} x(1)+c_{22} x^{\prime}(1)=0,
$$

where $c_{i j}$ are constants such that $c_{11}^{2}+c_{12}^{2}=c_{21}^{2}+c_{22}^{2}=1$. By an eigenvalue $\lambda$ of problem (1.1)-(1.2) is meant that with such a parameter $\lambda$, equation (1.1) has a non-zero solution $x(t)$ satisfying the conditions in (1.2). It is well known that all eigenvalues of problem (1.1)-(1.2) are given by a sequence

$$
\lambda_{0}(q)<\lambda_{1}(q)<\cdots<\lambda_{m}(q)<\cdots, \quad \lim _{m \rightarrow \infty} \lambda_{m}(q)=+\infty .
$$

Here $\lambda_{0}(q)$ may be void for some boundary conditions. See, for example, [4, 28, 29]. The eigenvalues $\lambda_{m}(q)$ are also dependent on the exponent $p$ and the boundary data $c_{i j}$.

Received by the editors September 12, 2008 and, in revised form, March 17, 2009.

2010 Mathematics Subject Classification. Primary 47J10, 34L15; Secondary 58C07, 58C40.

Key words and phrases. Eigenvalue, $p$-Laplacian, potential, weight, continuity, weak topology, differentiability, extremal value.

This research was supported by the National Basic Research Program of China (Grant no. 2006CB805903), the National Natural Science Foundation of China (Grant no. 10531010) and the 111 Project of China (2007).

(C)2010 American Mathematical Society Reverts to public domain 28 years from publication 
In this paper we will study the dependence of the eigenvalues $\lambda_{m}(q)$ on the potentials $q$. As usual, one typical topology on the space $\mathcal{L}^{\gamma}$ of potentials is the $L^{\gamma}$ topology induced by the $L^{\gamma}$ norm, denoted simply by $\|\cdot\|_{\gamma}=\|\cdot\|_{L^{\gamma}[0,1]}$. Besides this, we will also consider the weak topologies in these spaces. More precisely, for $\gamma \in[1, \infty)$, we use $w_{\gamma}$ to indicate the topology of weak convergence in the Banach space $\left(\mathcal{L}^{\gamma},\|\cdot\|_{\gamma}\right)$, and for $\gamma=\infty$, by considering the space $\left(\mathrm{E}^{\infty},\|\cdot\|_{\infty}\right)$ as the conjugate space of the Banach space $\left(\mathrm{E}^{1},\|\cdot\|_{1}\right)$, one has the topology $w_{\infty}$ induced by the weak* convergence in $\left(\mathrm{七}^{\infty},\|\cdot\|_{\infty}\right)$. In a unified way, $g_{n} \rightarrow g_{0}$ in $\left(\mathcal{L}^{\gamma}, w_{\gamma}\right)$ if and only if

$$
\int_{0}^{1} g_{n}(t) f(t) d t \rightarrow \int_{0}^{1} g_{0}(t) f(t) d t
$$

for each $f(t) \in \mathrm{E}^{\gamma^{*}}$. Here $\gamma^{*}:=\gamma /(\gamma-1)$ is the conjugate exponent of $\gamma$. See [27, Ch. 38].

For eigenvalues of the $p$-Laplacian with potentials, we can establish two fundamental results. The first one is the continuity of eigenvalues in weak topologies.

Theorem 1.1. Given $\gamma \in[1, \infty]$ and an admissible $m$, the following functional is continuous:

$$
\left(\mathcal{L}^{\gamma}, w_{\gamma}\right) \rightarrow \mathbb{R}, \quad q \rightarrow \lambda_{m}(q)
$$

This theorem is a generalization of the results in [24, 30]. See also [22] for some related results on eigenvalues of Dirac operators.

The second result is the differentiability (in the sense of Fréchet) of eigenvalues in potentials with the $L^{\gamma}$ norms.

Theorem 1.2. Given $\gamma \in[1, \infty]$ and an admissible $m$, the functional

$$
\left(\mathcal{L}^{\gamma},\|\cdot\|_{\gamma}\right) \rightarrow \mathbb{R}, \quad q \rightarrow \lambda_{m}(q)
$$

is continuously differentiable. Moreover, at any $q \in \mathcal{L}^{\gamma}$, the differential of $\lambda_{m}(q)$, denoted by $\partial_{q} \lambda_{m}(q) \in\left(\mathcal{L}^{\gamma},\|\cdot\|_{\gamma}\right)^{*}$, is given by

$$
\partial_{q} \lambda_{m}(q)(t)=-\left|E_{m}(t ; q)\right|^{p}, \quad t \in[0,1] .
$$

Here $E_{m}(t ; q)$ is the eigenfunction associated with $\lambda_{m}(q)$ satisfying the normalization condition

$$
\left\|E_{m}(\cdot ; q)\right\|_{p}=\left(\int_{0}^{1}\left|E_{m}(t ; q)\right|^{p} d t\right)^{1 / p}=1,
$$

while the function $-\left|E_{m}(\cdot ; q)\right|^{p}$ of (1.3) is understood as

$$
-\left|E_{m}(\cdot ; q)\right|^{p} \in\left(E^{\gamma^{*}},\|\cdot\|_{\gamma^{*}}\right) \subset\left(\mathcal{L}^{\gamma},\|\cdot\|_{\gamma}\right)^{*} \text {. }
$$

The differentiability result is a generalization of the works [23] where the differentiability of simple eigenvalues of linear differential operators is proved using an approach different from here. It is interesting to notice that formula (1.3) for differentials of eigenvalues can be expressed simply by using the associated eigenfunctions.

Consider the scalar equation

$$
\left(\phi_{p}\left(x^{\prime}\right)\right)^{\prime}+q(t) \phi_{p}(x)=0, \quad t \in[0,1] .
$$

Let $y=-\phi_{p}\left(x^{\prime}\right)$ and introduce the following $p$-polar coordinates:

$$
x=r^{2 / p} C_{p}(\theta), \quad y=r^{2 / p^{*}} S_{p}(\theta),
$$


where $C_{p}(\theta)$ and $S_{p}(\theta)$ are the so-called $p$-cosine and $p$-sine functions whose properties will be collected in Lemma 2.1. In order to prove Theorems 1.1 and 1.2, we will mainly study in Section 2 the dependence of $\theta=\theta(t ; \vartheta, q)$, the argument of equation (1.5), on the potential $q$. It will be proved that the argument $\theta(t ; \vartheta, q)$ is continuous in $q$ with respect to $w_{\gamma}$ and is continuously differentiable in $q$ with respect to $\|\cdot\|_{\gamma}$. See Theorem 2.3 and Theorem 2.5 , respectively.

The complete proofs of Theorems 1.1 and 1.2 will be given in Section 3 , In this section, by considering $q \in \mathcal{L}^{\gamma}$ as 1-periodic potentials, we will also prove that the rotation number $\varrho(q)$ of (1.5) (see [29]) and the variational 1-periodic and 1anti-periodic eigenvalues of (1.1) (see [5, 6]) are also continuous in $q \in\left(\mathcal{L}^{\gamma}, w_{\gamma}\right)$. See Theorem 3.5 and Theorem 3.6 respectively. It is well known that even for the linear case $p=2$, the latter objects are not continuously differentiable at all $q \in\left(\mathcal{L}^{\gamma},\|\cdot\|_{\gamma}\right)$. In this sense, the results in Theorems 1.1 and 1.2 are optimal.

In Section 4, taking the Dirichlet problem as an example, we will give a partial generalization of Theorems 1.1 and 1.2 to weighted eigenvalues of the onedimensional $p$-Laplacian

$$
\left(\phi_{p}\left(x^{\prime}\right)\right)^{\prime}+\mu \omega(t) \phi_{p}(x)=0, \quad t \in[0,1],
$$

with the Dirichlet boundary condition

$$
x(0)=x(1)=0 .
$$

Here the weight $\omega \in \mathcal{L}^{\gamma} \backslash\{0\}$. When the weight $\omega=\rho$ is (positive-) definite, i.e.,

$$
\rho \in \mathcal{W}^{\gamma}:=\left\{\rho \in \mathcal{L}^{\gamma}: \rho(t) \geq 0 \text { a.e. } t \in[0,1] \text {, and } \int_{0}^{1} \rho(t) d t>0\right\},
$$

it is well known [15, 28, that the eigenvalues of (1.7)-(1.8) are a sequence $\left\{\mu_{m}(\rho)\right\}_{m \in \mathbb{N}}$ of positive numbers. We will show that the $\mu_{m}(\rho)$ are also continuous in $\rho \in$ $\left(\mathcal{W}^{\gamma}, w_{\gamma}\right)$. See Theorem 4.2. Notice that for any $\gamma \in[1, \infty], \mathcal{W}^{\gamma} \subset\left(\mathcal{L}^{\gamma},\|\cdot\|_{\gamma}\right)$ is not open because of the restriction $\rho(t) \geq 0$ for a.e. $t \in[0,1]$. However, we can apply the Implicit Function Theorem to prove that $\left.\mu_{m}\right|_{\mathcal{W} \gamma}$ can be locally extended to a continuously differentiable functional. See Theorem 4.3 .

We think the results of this paper will be important in many problems concerned with eigenvalues. As an example, in Section 5, we will present an application of the results of this paper to a basic extremal problem of weighted eigenvalues. Roughly speaking, the continuity of eigenvalues in weak topologies can yield the existence of minimizers and maximizers if only the domains are compact in weak topologies, while differentials of eigenvalues can be used to deduce the critical equations for minimal and maximal potentials or weights, as done in 31] for the smallest periodic eigenvalues of linear Hill's equations. Compared with the traditional approach to extremal problems of eigenvalues in [16, 18, 20, 25, the approach here is quite different and is easy to handle. Some further applications to extremal problems of eigenvalues will be undertaken in future works.

\section{The $p$-POlar COORDinAtes AND THE ARGUMentS}

Given an exponent $p \in(1, \infty)$ and a potential $q \in \mathcal{L}^{\gamma}, \gamma \in[1, \infty]$, let us consider the scalar equation (1.5). By setting $y=-\phi_{p}\left(x^{\prime}\right)$, equation (1.5) can be rewritten as a planar system

$$
x^{\prime}=-\phi_{p^{*}}(y), \quad y^{\prime}=q(t) \phi_{p}(x) .
$$


In the $(x, y)$-plane, let us introduce the $p$-polar coordinates (1.6), where $\left(C_{p}(\theta)\right.$, $\left.S_{p}(\theta)\right), \theta \in \mathbb{R}$, is the unique solution of

$$
\frac{d x}{d \theta}=-\phi_{p^{*}}(y), \quad \frac{d y}{d \theta}=\phi_{p}(x),
$$

satisfying $(x(0), y(0))=(1,0)$. These functions $C_{p}(\theta)$ and $S_{p}(\theta)$ are the so-called $p$-cosine and $p$-sine because they possess properties similar to those of the standard cosine and sine as shown in the following lemma.

Lemma 2.1. The p-cosine and the p-sine have the following properties.

(i) Both $C_{p}(\theta)$ and $S_{p}(\theta)$ are $2 \pi_{p}$-periodic, where

$$
\pi_{p}=\frac{2 \pi(p-1)^{1 / p}}{p \sin (\pi / p)}
$$

(ii) $C_{p}(\theta)$ is even in $\theta$ and $S_{p}(\theta)$ is odd in $\theta$;

(iii) $C_{p}\left(\theta+\pi_{p}\right)=-C_{p}(\theta), S_{p}\left(\theta+\pi_{p}\right)=-S_{p}(\theta)$;

(iv) $C_{p}(\theta)=0$ if and only if $\theta=\pi_{p} / 2+n \pi_{p}, n \in \mathbb{Z}$, and $S_{p}(\theta)=0$ if and only if $\theta=n \pi_{p}, n \in \mathbb{Z}$;

(v) $\frac{d}{d \theta} C_{p}(\theta)=-\phi_{p^{*}}\left(S_{p}(\theta)\right)$ and $\frac{d}{d \theta} S_{p}(\theta)=\phi_{p}\left(C_{p}(\theta)\right)$;

(vi) $\left|C_{p}(\theta)\right|^{p}+(p-1)\left|S_{p}(\theta)\right|^{p^{*}} \equiv 1$.

In the $p$-polar coordinates (1.6), system (2.1) is transformed into the following equations for $r$ and $\theta$ :

$$
\begin{aligned}
(\log r)^{\prime} & =G(t, \theta ; q):=(p / 2)(q(t)-1) \phi_{p}\left(C_{p}(\theta)\right) \phi_{p^{*}}\left(S_{p}(\theta)\right) \\
\theta^{\prime} & =A(t, \theta ; q):=q(t)\left|C_{p}(\theta)\right|^{p}+(p-1)\left|S_{p}(\theta)\right|^{p^{*}} .
\end{aligned}
$$

Notice that $A(t, \theta ; q)$ is independent of $r$. Moreover, both $G(t, \theta ; q)$ and $A(t, \theta ; q)$ are $\pi_{p}$-periodic in $\theta$, and $A(t, \theta ; q)$ is continuously differentiable in $\theta$ :

$$
\frac{\partial A(t, \theta ; q)}{\partial \theta} \equiv-2 G(t, \theta ; q) .
$$

For the detailed constructions, one can refer to 29 .

In the following we first consider equation (2.3) for the argument $\theta$. For any $\vartheta \in \mathbb{R}$, denote by $\theta(t ; \vartheta, q), t \in[0,1]$, the unique solution of (2.3) satisfying the initial condition $\theta(0 ; \vartheta, q)=\vartheta$.

The solution $\theta(\cdot ; \vartheta, q)$ is a function in the space $\mathcal{C}^{0}:=C([0,1], \mathbb{R})$ of continuous functions from $[0,1]$ to $\mathbb{R}$. Suppose that $\vartheta \in \mathbb{R}$ is fixed. It is well known that the solution $\theta(\cdot ; \vartheta, q)$ depends on $q \in \mathcal{L}^{\gamma}$ in a continuous way when the usual $L^{\gamma}$ topology $\|\cdot\|_{\gamma}$ on $\mathcal{L}^{\gamma}$ is considered. In this paper, in order to prove Theorem 1.1. we will actually show that $\theta(\cdot ; \vartheta, q)$ has a stronger continuity in the potential $q$. From the characterization of sequentially compact subsets in the space $\left(\mathrm{E}^{1}, w_{1}\right)$ (see [10, p. 294]), one has the following important observation on weak convergence in $\left(\mathrm{E}^{1}, w_{1}\right)$.

Lemma $2.2([22])$. Let $\left\{f_{n}\right\}_{n \geq 0} \subset E^{1}$ be such that $f_{n} \rightarrow f_{0}$ in $\left(E^{1}, w_{1}\right)$ and $\left\{g_{n}\right\}_{n \geq 0} \subset t^{\infty}$. Set

$$
h_{n}(t):=\int_{0}^{t} f_{n}(s) g_{n}(s) d s, \quad t \in[0,1] .
$$


It is obvious that $\left\{h_{n}\right\}_{n \geq 0} \subset \mathcal{C}^{0}$. We have the following conclusions.

(i) If $\left\{g_{n}\right\}_{n \geq 1} \subset\left(E^{\infty},\|\cdot\|_{\infty}\right)$ is bounded, then $\left\{h_{n}\right\}_{n \geq 1} \subset\left(\mathcal{C}^{0},\|\cdot\|_{\infty}\right)$ is a relatively sequentially compact subset.

(ii) If $g_{n} \rightarrow g_{0}$ in $\left(E^{\infty},\|\cdot\|_{\infty}\right)$, then $h_{n} \rightarrow h_{0}$ in $\left(\mathcal{C}^{0},\|\cdot\|_{\infty}\right)$.

Now we can give one of the main results of this section on arguments.

Theorem 2.3. Let $\gamma \in[1, \infty]$ and $\vartheta \in \mathbb{R}$. The following solution mapping of equation (2.3) is continuous:

$$
\left(\mathcal{L}^{\gamma}, w_{\gamma}\right) \rightarrow\left(\mathcal{C}^{0},\|\cdot\|_{\infty}\right), \quad q \rightarrow \theta(\cdot ; \vartheta, q) .
$$

Proof. At first, let us notice that if $\gamma \in(1, \infty]$, then one has

$$
q_{n} \rightarrow q_{0} \text { in }\left(\mathcal{L}^{\gamma}, w_{\gamma}\right) \Longrightarrow q_{n} \rightarrow q_{0} \text { in }\left(\mathrm{E}^{1}, w_{1}\right) .
$$

Thus it suffices to prove the theorem only for the case $\gamma=1$.

Let $\left\{q_{n}\right\}_{n \geq 0} \subset \mathrm{E}^{1}$ be such that $q_{n} \rightarrow q_{0}$ in $\left(\mathrm{E}^{1}, w_{1}\right)$. Denote

$$
\theta_{n}(t):=\theta\left(t ; \vartheta, q_{n}\right) \in \mathcal{C}^{0}, \quad n \geq 0 .
$$

We need to prove that $\theta_{n} \rightarrow \theta_{0}$ in $\left(\mathcal{C}^{0},\|\cdot\|_{\infty}\right)$.

Step 1. Since $\theta_{n}(t)$ is the solution of equation (2.3) with $q=q_{n}$, we get

$$
\begin{aligned}
\theta_{n}(t) & =\vartheta+\int_{0}^{t} q_{n}(s)\left|C_{p}\left(\theta_{n}(s)\right)\right|^{p} d s+\int_{0}^{t}(p-1)\left|S_{p}\left(\theta_{n}(s)\right)\right|^{p^{*}} d s \\
& =: \quad \vartheta+\theta_{n}^{1}(t)+\theta_{n}^{2}(t), \quad t \in[0,1],
\end{aligned}
$$

where, for $n=0,1,2, \ldots$,

$$
\begin{aligned}
& \theta_{n}^{1}(t)=\int_{0}^{t} q_{n}(s)\left|C_{p}\left(\theta_{n}(s)\right)\right|^{p} d s, \\
& \theta_{n}^{2}(t)=\int_{0}^{t}(p-1)\left|S_{p}\left(\theta_{n}(s)\right)\right|^{p^{*}} d s .
\end{aligned}
$$

Step 2. Now we are going to apply Lemma 2.2 (i) to (2.7) and (2.8). For the sequence (2.7), let $f_{n}(t)=q_{n}(t), n \geq 0$, and $g_{n}(t)=\left|C_{p}\left(\theta_{n}(t)\right)\right|^{p}, n \geq 1$. We know that $f_{n} \rightarrow f_{0}$ in $\left(\mathrm{E}^{1}, w_{1}\right)$ and $\left\{g_{n}\right\}_{n \geq 1} \subset\left(\mathrm{E}^{\infty},\|\cdot\|_{\infty}\right)$ is bounded. Thus $\left\{\theta_{n}^{1}\right\}_{n \geq 1} \subset\left(\mathcal{C}^{0},\|\cdot\|_{\infty}\right)$ is relatively (sequentially) compact. Similarly, by setting $f_{n}(t) \equiv p-1, n \geq 0$ and $g_{n}(t)=\left|S_{p}\left(\theta_{n}(t)\right)\right|^{p^{*}}, n \geq 1$, we know that $\left\{\theta_{n}^{2}\right\}_{n \geq 1} \subset$ $\left(\mathcal{C}^{0},\|\cdot\|_{\infty}\right)$ is also relatively compact. In conclusion, by formula (2.6), the sequence $\left\{\theta_{n}\right\}_{n \geq 1} \subset\left(\mathcal{C}^{0},\|\cdot\|_{\infty}\right)$ is relatively compact.

Step 3. Let $\left\{\theta_{n^{\prime}}\right\}$ be any subsequence of $\left\{\theta_{n}\right\}_{n \geq 1}$. From Step 2, one has a subsubsequence $\left\{\theta_{n^{\prime \prime}}\right\}$ of $\left\{\theta_{n^{\prime}}\right\}$ such that, as $n^{\prime \prime} \rightarrow \infty$, it follows that

$$
\theta_{n^{\prime \prime}} \rightarrow \theta_{*} \text { in }\left(\mathcal{C}^{0},\|\cdot\|_{\infty}\right) \text { for some } \theta_{*} \in \mathcal{C}^{0}
$$

As $C_{p}$ and $S_{p}$ are continuous, the corresponding sequences $\left\{\left|C_{p}\left(\theta_{n^{\prime \prime}}(t)\right)\right|^{p}\right\}$ and $\left\{\left|S_{p}\left(\theta_{n^{\prime \prime}}(t)\right)\right|^{p^{*}}\right\}$ converge to $\left|C_{p}\left(\theta_{*}(t)\right)\right|^{p}$ and $\left|S_{p}\left(\theta_{*}(t)\right)\right|^{p^{*}}$ in $\left(\mathcal{C}^{0},\|\cdot\|_{\infty}\right)$, respectively. Now applying Lemma 2.2 (ii) to these sub-subsequences, we know from the right-hand sides of (2.5) that

$$
\theta_{n^{\prime \prime}}(t) \rightarrow \vartheta+\int_{0}^{t} q_{0}(s)\left|C_{p}\left(\theta_{*}(s)\right)\right|^{p} d s+\int_{0}^{t}(p-1)\left|S_{p}\left(\theta_{*}(s)\right)\right|^{p^{*}} d s
$$


in $\left(\mathcal{C}^{0},\|\cdot\|_{\infty}\right)$. From the uniqueness of the limit of $\left\{\theta_{n^{\prime \prime}}\right\}$, by (2.9) and (2.10), the limit $\theta_{*}(t)$ satisfies

$$
\theta_{*}(t) \equiv \vartheta+\int_{0}^{t} q_{0}(s)\left|C_{p}\left(\theta_{*}(s)\right)\right|^{p} d s+\int_{0}^{t}(p-1)\left|S_{p}\left(\theta_{*}(s)\right)\right|^{p^{*}} d s, \quad t \in[0,1] .
$$

This shows that $\theta_{*}(t)$ is just the solution of (2.3) with $q=q_{0}$. We conclude that

$$
\theta_{*}(t) \equiv \theta_{0}(t)=\theta\left(t ; \vartheta, q_{0}\right) .
$$

Step 4. Equality (2.11) shows that the limit $\theta_{0} \in \mathcal{C}^{0}$ is independent of the choice of subsequences $\left\{\theta_{n^{\prime}}\right\}$ and $\left\{\theta_{n^{\prime \prime}}\right\}$. Consequently, the sequence $\left\{\theta_{n}\right\}_{n \geq 1}$ itself is convergent to $\theta_{0}$ in $\left(\mathcal{C}^{0},\|\cdot\|_{\infty}\right)$, proving the theorem.

Remark 2.4. (i) By similar arguments as in the proof of Theorem 2.3. we can obtain a little bit stronger continuity result. That is, the following mapping,

$$
\mathbb{R} \times\left(\mathcal{L}^{\gamma}, w_{\gamma}\right) \rightarrow\left(\mathcal{C}^{0},\|\cdot\|_{\infty}\right), \quad(\vartheta, q) \rightarrow \theta(\cdot ; \vartheta, q),
$$

is jointly continuous in $(\vartheta, q)$.

(ii) Notice that the solutions $\theta(t ; \vartheta, q)=\theta(t ; \vartheta, q, p)$ are also dependent on the exponent $p$. If we consider the exponent $p$ as another parameter, then

$$
\mathbb{R} \times\left(\mathcal{L}^{\gamma}, w_{\gamma}\right) \times(1, \infty) \rightarrow\left(\mathcal{C}^{0},\|\cdot\|_{\infty}\right), \quad(\vartheta, q, p) \rightarrow \theta(\cdot ; \vartheta, q, p),
$$

is also jointly continuous in $(\vartheta, q, p)$.

Now we can give the continuous differentiability of solutions $\theta(t ; \vartheta, q)$ in potentials $q$ with respect to the $L^{\gamma}$ norms. Without loss of generality, we only consider solutions at $t=1$. Given $\vartheta \in \mathbb{R}$, let us define the solution functional $\Theta: \mathcal{L}^{\gamma} \rightarrow \mathbb{R}$ of equation (2.3) by

$$
\Theta(\vartheta, q):=\theta(1 ; \vartheta, q) .
$$

Theorem 2.5. Let $\gamma \in[1, \infty]$ and $\vartheta \in \mathbb{R}$ be fixed.

(i) The following functional is continuously differentiable:

$$
\left(\mathcal{L}^{\gamma},\|\cdot\|_{\gamma}\right) \rightarrow \mathbb{R}, \quad q \rightarrow \Theta(\vartheta, q) .
$$

(ii) Define

$$
\begin{aligned}
X(t ; \vartheta, q)= & \exp \left(\int_{1}^{t}(q(\tau)-1) \phi_{p}\left(C_{p}(\theta(\tau ; \vartheta, q))\right) \phi_{p^{*}}\left(S_{p}(\theta(\tau ; \vartheta, q))\right) d \tau\right) \\
& \times C_{p}(\theta(t ; \vartheta, q)), \quad t \in[0,1] .
\end{aligned}
$$

Then $X(\cdot ; \vartheta, q)$ is a solution of equation (1.5). Moreover, the differential of $\Theta(\vartheta, q)$ at $q \in \mathcal{L}^{\gamma}$, denoted by $\partial_{q} \Theta(\vartheta, q)$, is the following function:

$$
\partial_{q} \Theta(\vartheta, q)(t)=|X(t ; \vartheta, q)|^{p} \in \mathcal{C}^{0} \subset\left(\mathcal{L}^{\gamma},\|\cdot\|_{\gamma}\right)^{*} .
$$

Proof. Step 1. Let $q \in \mathcal{L}^{\gamma}$ be fixed. We first compute the directional derivative of $\Theta(\vartheta, q)$ along the direction $h \in \mathcal{L}^{\gamma}$, which is defined as

$$
D_{q}(h)=\left.\frac{d \Theta(\vartheta, q+\varepsilon h)}{d \varepsilon}\right|_{\varepsilon=0} .
$$

For simplicity, let us write

$$
\theta(t):=\theta(t ; \vartheta, q), \quad \theta(t, \varepsilon):=\theta(t ; \vartheta, q+\varepsilon h), \quad t \in[0,1] .
$$


Notice that $\theta(0, \varepsilon) \equiv \vartheta$. Moreover, from (2.3) we know that $\theta(t, \varepsilon)$ satisfies

$$
\frac{d}{d t} \theta(t, \varepsilon)=(q(t)+\varepsilon h(t))\left|C_{p}(\theta(t, \varepsilon))\right|^{p}+(p-1)\left|S_{p}(\theta(t, \varepsilon))\right|^{p^{*}} .
$$

Thus $\theta(t, \varepsilon)$ is continuously differentiable in $\varepsilon$. For simplicity, let us denote

$$
\psi(t):=\left.\frac{d \theta(t, \varepsilon)}{d \varepsilon}\right|_{\varepsilon=0} .
$$

Thus $\psi(0)=0$ because $\theta(0, \varepsilon) \equiv \vartheta$. Moreover, by differentiating (2.17) with respect to $\varepsilon$ at $\varepsilon=0$, we have the following variational equation for $\psi(t)$ :

$$
\frac{d}{d t} \psi(t)=h(t)\left|C_{p}(\theta(t))\right|^{p}+p a(t) \psi(t) .
$$

Here

$$
a(t):=-(2 / p) G(t, \theta(t) ; q)=-(q(t)-1) \phi_{p}\left(C_{p}(\theta(t))\right) \phi_{p^{*}}\left(S_{p}(\theta(t))\right) .
$$

See (2.2) -(2.4) and the notation for $\theta(t)$ in (2.16). As $\psi(0)=0$, the unique solution of the linear equation (2.18) is

$$
\psi(t)=\int_{0}^{t} \exp \left(\int_{s}^{t} p a(\tau) d \tau\right)\left|C_{p}(\theta(s))\right|^{p} \cdot h(s) d s, \quad t \in[0,1] .
$$

In particular,

$$
\begin{aligned}
D_{q}(h) & =\int_{0}^{1} \exp \left(\int_{s}^{1} p a(\tau) d \tau\right)\left|C_{p}(\theta(s))\right|^{p} \cdot h(s) d s \\
& =\int_{0}^{1}|X(s ; \vartheta, q)|^{p} \cdot h(s) d s,
\end{aligned}
$$

where $X(t ; \vartheta, q)$ is as in (2.14). Since

$$
|X(\cdot ; \vartheta, q)|^{p} \in \mathcal{C}^{0} \subset\left(\mathrm{E}^{\gamma^{*}},\|\cdot\|_{\gamma^{*}}\right) \subset\left(\mathcal{L}^{\gamma},\|\cdot\|_{\gamma}\right)^{*},
$$

$D_{q}(h)$ of (2.20) defines a bounded linear functional $D_{q} \in\left(\mathcal{L}^{\gamma},\|\cdot\|_{\gamma}\right)^{*}$.

Step 2. In order to prove the continuous differentiability of $\Theta(\vartheta, q)$ in the potential $q \in\left(\mathcal{L}^{\gamma},\|\cdot\|_{\gamma}\right)$, it suffices to prove that the bounded linear functional $D_{q}$ is continuous in $q$. More precisely, we need only to prove that the following mapping,

$$
\left(\mathcal{L}^{\gamma},\|\cdot\|_{\gamma}\right) \rightarrow\left(\mathrm{E}^{\gamma^{*}},\|\cdot\|_{\gamma^{*}}\right), \quad q \rightarrow|X(\cdot ; \vartheta, q)|^{p},
$$

is continuous. In Corollary 2.6 below, we will prove a stronger continuity result. Now (2.20) can be explained as the equality (2.15) for the differential of $\Theta(\vartheta, q)$ in $q$.

Step 3. Finally, let us prove that $X(t ; \vartheta, q)$ is a solution of equation (1.5). Recall that $\theta(t ; \vartheta, q)$ is a solution of (2.3). It follows from equation (2.2) that

$$
r(t ; \vartheta, q):=\exp \left(\int_{1}^{t} G(\tau, \theta(\tau ; \vartheta, q) ; q) d \tau\right)
$$

is a solution of (2.2) satisfying $r(1 ; \vartheta, q)=1$. Let us write the first factor of (2.14) as

$$
R(t ; \vartheta, q):=\exp \left(\int_{1}^{t}(q(\tau)-1) \phi_{p}\left(C_{p}(\theta(\tau ; \vartheta, q))\right) \phi_{p^{*}}\left(S_{p}(\theta(\tau ; \vartheta, q))\right) d \tau\right) .
$$

By (2.19), we have the equality

$$
R(t ; \vartheta, q) \equiv(r(t ; \vartheta, q))^{2 / p} .
$$


Now the function $X(t ; \vartheta, q)$ of (2.14) can be rewritten as

$$
X(t ; \vartheta, q)=R(t ; \vartheta, q) C_{p}(\theta(t ; \vartheta, q))=(r(t ; \vartheta, q))^{2 / p} C_{p}(\theta(t ; \vartheta, q)) .
$$

Using the $p$-polar coordinates (1.6), we know that $X(t ; \vartheta, q)$ is a solution of equation (1.5).

Corollary 2.6. Given $\gamma \in[1, \infty]$ and $\vartheta \in \mathbb{R}$, the following mappings are continuous:

$$
\begin{array}{lll}
\left(\mathcal{L}^{\gamma}, w_{\gamma}\right) \rightarrow\left(\mathcal{C}^{0},\|\cdot\|_{\infty}\right), & & q X X(\cdot ; \vartheta, q), \\
\left(\mathcal{L}^{\gamma}, w_{\gamma}\right) \rightarrow\left(\mathcal{C}^{0},\|\cdot\|_{\infty}\right), & & q|X(\cdot ; \vartheta, q)|^{p} .
\end{array}
$$

Proof. It suffices to prove (2.23), because the continuity of (2.24) is simply a consequence of (2.23). Let $q_{n} \rightarrow q_{0}$ in $\left(\mathcal{L}^{\gamma}, w_{\gamma}\right)$. By Theorem 2.3, we have, in $\left(\mathcal{C}^{0},\|\cdot\|_{\infty}\right)$,

$$
\theta\left(\cdot ; \vartheta, q_{n}\right) \rightarrow \theta\left(\cdot ; \vartheta, q_{0}\right), \quad C_{p}\left(\theta\left(\cdot ; \vartheta, q_{n}\right)\right) \rightarrow C_{p}\left(\theta\left(\cdot ; \vartheta, q_{0}\right)\right) .
$$

By the first formula of (2.22), we need only to prove

$$
R\left(\cdot ; \vartheta, q_{n}\right) \rightarrow R\left(\cdot ; \vartheta, q_{0}\right) \quad \text { in }\left(\mathcal{C}^{0},\|\cdot\|_{\infty}\right) .
$$

Notice that

$$
\begin{aligned}
f_{n}(t) & :=q_{n}(t)-1 \rightarrow q_{0}(t)-1=: f_{0}(t) \quad \text { in }\left(\mathrm{E}^{1}, w_{1}\right) \\
g_{n}(t) & :=\phi_{p}\left(C_{p}\left(\theta\left(t ; \vartheta, q_{n}\right)\right)\right) \phi_{p^{*}}\left(S_{p}\left(\theta\left(t ; \vartheta, q_{n}\right)\right)\right) \\
& \rightarrow \phi_{p}\left(C_{p}\left(\theta\left(t ; \vartheta, q_{0}\right)\right)\right) \phi_{p^{*}}\left(S_{p}\left(\theta\left(t ; \vartheta, q_{0}\right)\right)\right)=: g_{0}(t) \quad \text { in }\left(\mathcal{C}^{0},\|\cdot\|_{\infty}\right) .
\end{aligned}
$$

By Lemma 2.2 (ii), we have

$$
\begin{aligned}
\int_{1}^{t} f_{n}(s) g_{n}(s) d s & =\int_{0}^{t} f_{n}(s) g_{n}(s) d s-\int_{0}^{1} f_{n}(s) g_{n}(s) d s \\
& \rightarrow \int_{0}^{t} f_{0}(s) g_{0}(s) d s-\int_{0}^{1} f_{0}(s) g_{0}(s) d s \\
& =\int_{1}^{t} f_{0}(s) g_{0}(s) d s \quad \text { in }\left(\mathcal{C}^{0},\|\cdot\|_{\infty}\right) .
\end{aligned}
$$

By formula (2.21), one has

$$
R\left(t ; \vartheta, q_{n}\right)=\exp \left(\int_{1}^{t} f_{n}(s) g_{n}(s) d s\right) \rightarrow \exp \left(\int_{1}^{t} f_{0}(s) g_{0}(s) d s\right)=R\left(t ; \vartheta, q_{0}\right)
$$

in $\left(\mathcal{C}^{0},\|\cdot\|_{\infty}\right)$, proving the desired result (2.25).

Let $q_{i} \in \mathcal{L}^{\gamma}, i=0,1$. We write $q_{1} \geq q_{0}$ if $q_{1}(t) \geq q_{0}(t)$ for a.e. $t \in[0,1]$. We write $q_{1} \succ q_{0}$ if $q_{1} \geq q_{0}$ and $\int_{0}^{1} q_{1}(t) d t>\int_{0}^{1} q_{0}(t) d t$.

Lemma $2.7([29)$. Let $\vartheta \in \mathbb{R}$. Then

$$
\begin{aligned}
& q_{0}, q_{1} \in \mathcal{L}^{\gamma}, q_{1} \geq q_{0} \Longrightarrow \Theta\left(\vartheta, q_{1}\right) \geq \Theta\left(\vartheta, q_{0}\right), \\
& q_{0}, q_{1} \in \mathcal{L}^{\gamma}, q_{1} \succ q_{0} \Longrightarrow \Theta\left(\vartheta, q_{1}\right)>\Theta\left(\vartheta, q_{0}\right) .
\end{aligned}
$$

These results were proved in 29] using the comparison result for solutions of the first-order ODEs (2.3). They can also be obtained from (2.15) because the differentials are positive functionals. 


\section{Eigenvalues of the $p$-Laplacian With potentials}

3.1. Equations for eigenvalues. It is well known that separated eigenvalues of (1.1)-(1.2) can be obtained by solving equations concerned with the arguments. To this end, let us write the separated boundary conditions (1.2) in the following way:

$$
\phi_{p^{*}}\left(S_{p}(\alpha)\right) x(0)+C_{p}(\alpha) x^{\prime}(0)=0, \quad \phi_{p^{*}}\left(S_{p}(\beta)\right) x(1)+C_{p}(\beta) x^{\prime}(1)=0,
$$

where the boundary data $\alpha, \beta$ can take in any interval of length $\pi_{p}$. In this paper, we assume that $\alpha, \beta \in\left[-\pi_{p} / 2, \pi_{p} / 2\right)$. For example, the Dirichlet boundary condition corresponds to $\alpha=\beta=-\pi_{p} / 2$.

In the $p$-polar coordinates (1.6), we have

$$
x(t)=(r(t))^{2 / p} C_{p}(\theta(t)), \quad x^{\prime}(t)=-(r(t))^{2 / p} \phi_{p^{*}}\left(S_{p}(\theta(t))\right) .
$$

Then the boundary conditions (3.1) can be rewritten as

$$
\theta(0)=\alpha+k \pi_{p}, \quad \theta(1)=\beta+l \pi_{p},
$$

where $k, l \in \mathbb{Z}$. In terms of $\Theta$ of (2.13),$\lambda \in \mathbb{R}$ is an eigenvalue of problem (1.1)-(3.1) if and only if $\lambda \in \mathbb{R}$ satisfies

$$
\Theta(\alpha, \lambda+q)=\beta+m \pi_{p}
$$

for some integer $m \in \mathbb{Z}$. The range of $m$ depends on the boundary data $\beta$. To describe this, some well-known properties of $\theta(t ; \vartheta, q)$ and $\Theta(\vartheta, q)$ are listed in the following lemmas.

Lemma 3.1. Given $q \in \mathcal{L}^{\gamma}$ and $\vartheta \in \mathbb{R}$, let $\theta(t)=\theta(t ; \vartheta, q)$ be the solution of (2.3). Then

$$
\theta(t) \geq-\pi_{p} / 2+m \pi_{p} \text { at } t \in[0,1) \Rightarrow \theta(s)>-\pi_{p} / 2+m \pi_{p} \forall s \in(t, 1] .
$$

Here $m \in \mathbb{Z}$. In particular, $\Theta(\vartheta, \lambda+q)>-\pi_{p} / 2$ for any $\vartheta \in\left[-\pi_{p} / 2, \pi_{p} / 2\right)$ and any $\lambda \in \mathbb{R}$.

Lemma 3.2. Given $q \in \mathcal{L}^{\gamma}$ and $\vartheta \in \mathbb{R}$, then

(i) $\Theta(\vartheta, \lambda+q)$ is strictly increasing in $\lambda \in \mathbb{R}$;

(ii) $\lim _{\lambda \rightarrow+\infty} \Theta(\vartheta, \lambda+q)=+\infty$;

(iii) $\lim _{\lambda \rightarrow-\infty} \Theta(\vartheta, \lambda+q)=-\pi_{p} / 2$ if $\vartheta \in\left[-\pi_{p} / 2, \pi_{p} / 2\right)$.

Consequently, (3.2) has some solution $\lambda=\lambda_{m}(q ; \alpha, \beta)$ when and only when

$$
m \in N_{\beta}:= \begin{cases}\mathbb{N} & \text { if } \beta=-\pi_{p} / 2 \\ \mathbb{Z}^{+}:=\mathbb{N} \cup\{0\} & \text { otherwise. }\end{cases}
$$

Namely, all the eigenvalues of problem (1.1)-(3.1) consist of a sequence $\lambda=\lambda_{m}(q)=$ $\lambda_{m}(q ; \alpha, \beta), m \in N_{\beta}$. In particular, for the Dirichlet boundary conditions, the zeroth eigenvalues $\lambda_{0}(q)$ are meaningless. For detailed proofs of these results, see [4] and [5, Theorem 1.1].

\subsection{Continuity of eigenvalues and eigenfunctions in weak topologies.}

Proof of Theorem [1.1. Let $\alpha, \beta$ and $m \in N_{\beta}$ be specified. Suppose that $q_{n} \rightarrow q_{0}$ in $\left(\mathcal{L}^{\gamma}, w_{\gamma}\right)$. Denote $\nu_{n}:=\lambda_{m}\left(q_{n} ; \alpha, \beta\right)$. One has

$$
\Theta\left(\alpha, \nu_{n}+q_{n}\right)=\beta+m \pi_{p}, \quad n \in \mathbb{Z}^{+} .
$$


We need to prove that $\nu_{n} \rightarrow \nu_{0}$ as $n \rightarrow \infty$. If not, passing to a subsequence, we assume that there exists $\varepsilon_{0}>0$ such that

$$
\left|\nu_{n}-\nu_{0}\right| \geq \varepsilon_{0} \quad \forall n \geq 1 .
$$

Without loss of generality, we may assume further that

$$
\nu_{n} \geq \nu_{0}+\varepsilon_{0} \quad \forall n \geq 1 .
$$

Thus

$$
\nu_{n}+q_{n} \geq \nu_{0}+\varepsilon_{0}+q_{n} .
$$

By the monotonicity in Lemma 2.7, we obtain from (3.3) and (3.4) that

$$
\beta+m \pi_{p}=\Theta\left(\alpha, \nu_{n}+q_{n}\right) \geq \Theta\left(\alpha, \nu_{0}+\varepsilon_{0}+q_{n}\right) \quad \forall n \geq 1 .
$$

Letting $n \rightarrow \infty$ in (3.5) and noticing that $\nu_{0}+\varepsilon_{0}+q_{n} \rightarrow \nu_{0}+\varepsilon_{0}+q_{0}$ in $\left(\mathcal{L}^{\gamma}, w_{\gamma}\right)$, we have

$$
\begin{aligned}
\beta+m \pi_{p} & \geq \Theta\left(\alpha, \nu_{0}+\varepsilon_{0}+q_{0}\right) \quad \text { (by Theorem 2.3) } \\
& >\Theta\left(\alpha, \nu_{0}+q_{0}\right) \quad(\text { by Lemma 2.7) } \\
& =\beta+m \pi_{p} \quad(\text { by }(\underline{3.3}) .
\end{aligned}
$$

Such a contradiction proves Theorem 1.1

Remark 3.3. Instead of Theorem 2.3 if we apply the results in Remark 2.4, we can actually prove that the eigenvalues $\lambda_{m}(q ; \alpha, \beta, p)$ are also continuous in the boundary data $(\alpha, \beta)$ and the exponent $p$. More precisely, the following mapping,

$$
\begin{gathered}
\left(\mathcal{L}^{\gamma}, w_{\gamma}\right) \times\left[-\pi_{p} / 2, \pi_{p} / 2\right) \times\left[-\pi_{p} / 2, \pi_{p} / 2\right) \times(1, \infty) \rightarrow \mathbb{R}, \\
(q, \alpha, \beta, p) \rightarrow \lambda_{m}(q ; \alpha, \beta, p),
\end{gathered}
$$

is jointly continuous in $(q, \alpha, \beta, p)$.

For any admissible $\alpha, \beta, m$, for simplicity, denote $\lambda_{m}(q):=\lambda_{m}(q ; \alpha, \beta)$. Let us define

$$
\begin{aligned}
& \hat{E}_{m}(t ; q)=\hat{E}_{m}(t ; q, \alpha, \beta):=X\left(t ; \alpha, \lambda_{m}(q)+q\right) \in \mathcal{C}^{0}, \\
& E_{m}(t ; q)=E_{m}(t ; q, \alpha, \beta):=\frac{\hat{E}_{m}(t ; q)}{\left\|\hat{E}_{m}(\cdot ; q)\right\|_{p}} \in \mathcal{C}^{0} .
\end{aligned}
$$

One knows from Theorem 2.5 (ii) that $x(t)=\hat{E}_{m}(t ; q)$ is a solution of

$$
\left(\phi_{p}\left(x^{\prime}\right)\right)^{\prime}+\left(\lambda_{m}(q)+q(t)\right) \phi_{p}(x)=0 .
$$

Moreover, combining (2.22) and (3.2), which determines $\lambda_{m}(q)$, one knows that $x(t)=\hat{E}_{m}(t ; q)$ satisfies the boundary condition (3.1). Thus $\hat{E}_{m}(t ; q)$ of (3.6) is an eigenfunction and $E_{m}(t ; q)$ of (3.7) is a normalized eigenfunction associated with $\lambda_{m}(q)$, respectively. These eigenfunctions $\hat{E}_{m}(t ; q)$ and $E_{m}(t ; q)$ also have a very strong continuous dependence on the potentials $q$.

Theorem 3.4. Given admissible $\alpha, \beta$ and $m$, both the eigenfunctions $\hat{E}_{m}(t ; q)$ and $E_{m}(t ; q)$ are continuous in $q \in\left(\mathcal{L}^{\gamma}, w_{\gamma}\right)$. More precisely, the following mappings are continuous:

$$
\left(\mathcal{L}^{\gamma}, w_{\gamma}\right) \rightarrow\left(\mathcal{C}^{0},\|\cdot\|_{\infty}\right), \quad q \rightarrow \hat{E}_{m}(\cdot ; q), \quad q \rightarrow E_{m}(\cdot ; q) .
$$


Proof. The proof combines the continuity results of Theorem 1.1] and Corollary 2.6.

Let us first consider $\hat{E}_{m}(\cdot ; q)$. Suppose that $q_{n} \rightarrow q_{0}$ in $\left(\mathcal{L}^{\gamma}, w_{\gamma}\right)$. By Theorem 1.1. we know that $\lambda_{m}\left(q_{n}\right) \rightarrow \lambda_{m}\left(q_{0}\right)$ in $\mathbb{R}$. Consequently,

$$
\tilde{q}_{n}:=\lambda_{m}\left(q_{n}\right)+q_{n} \rightarrow \lambda_{m}\left(q_{0}\right)+q_{0}=: \tilde{q}_{0} \quad \text { in }\left(\mathcal{L}^{\gamma}, w_{\gamma}\right) .
$$

By (2.23), we have, in $\left(\mathcal{C}^{0},\|\cdot\|_{\infty}\right)$,

$$
\hat{E}_{m}\left(\cdot ; q_{n}\right)=X\left(\cdot ; \alpha, \tilde{q}_{n}\right) \rightarrow X\left(\cdot ; \alpha, \tilde{q}_{0}\right)=\hat{E}_{m}\left(\cdot ; q_{0}\right) \quad \text { in }\left(\mathcal{C}^{0},\|\cdot\|_{\infty}\right) .
$$

Next, (3.8) implies that

$$
\left\|\hat{E}_{m}\left(\cdot ; q_{n}\right)\right\|_{p} \rightarrow\left\|\hat{E}_{m}\left(\cdot ; q_{0}\right)\right\|_{p} .
$$

Now (3.8) and (3.9) can yield

$$
E_{m}\left(\cdot ; q_{n}\right)=\frac{\hat{E}_{m}\left(\cdot ; q_{n}\right)}{\left\|\hat{E}_{m}\left(\cdot ; q_{n}\right)\right\|_{p}} \rightarrow \frac{\hat{E}_{m}\left(\cdot ; q_{0}\right)}{\left\|\hat{E}_{m}\left(\cdot ; q_{0}\right)\right\|_{p}}=E_{m}\left(\cdot ; q_{0}\right)
$$

in $\left(\mathcal{C}^{0},\|\cdot\|_{\infty}\right)$, which proves the continuity of $E_{m}(\cdot ; q)$ in $q \in\left(\mathcal{L}^{\gamma}, w_{\gamma}\right)$.

3.3. Differentiability of eigenvalues in $L^{\gamma}$ norms. Given admissible $\alpha, \beta$ and $m$, from (2.15) and (3.6), we have the equality

$$
\left.\partial_{q} \Theta(\alpha, \cdot)\right|_{\lambda_{m}(q)+q}=\left|\hat{E}_{m}(\cdot ; q)\right|^{p} .
$$

Proof of Theorem 1.2. Equation (3.2) for $\lambda=\lambda_{m}(q)$ can be rewritten as the following implicit function equation:

$$
F(\lambda, q)=\beta+m \pi_{p},
$$

where $F: \mathbb{R} \times\left(\mathcal{L}^{\gamma},\|\cdot\|_{\gamma}\right) \rightarrow \mathbb{R}$ is $F(\lambda, q):=\Theta(\alpha, \lambda+q)$. By Theorem 2.5. $F$ is continuously differentiable in $(\lambda, q)$. Moreover, the derivative of $F(\lambda, q)$ in $\lambda \in \mathbb{R}$ is simply

$$
\frac{d}{d \lambda} F(\lambda, q)=\int_{0}^{1}|X(s ; \alpha, \lambda+q)|^{p} d s>0 .
$$

See (2.20). Applying the Implicit Function Theorem to (3.11), we conclude that the solution function $\lambda=\lambda_{m}(q)$ of (3.11) is continuously differentiable in $q \in\left(\mathcal{L}^{\gamma},\|\cdot\|_{\gamma}\right)$.

Next let us give the differential $\partial_{q} \lambda_{m}(q)$. Let $q, h \in\left(\mathcal{L}^{\gamma},\|\cdot\|_{\gamma}\right)$. Write

$$
\lambda_{m}(q+\varepsilon h)=\lambda_{m}(q)+\varepsilon \ell(h)+o(\varepsilon) \quad \text { as } \varepsilon \rightarrow 0,
$$

where $\ell(h)=\partial_{q} \lambda_{m}(q) \cdot h \in \mathbb{R}$ is linear in $h$. By equation (3.11), we have

$$
\begin{aligned}
\beta+m \pi_{p} & \equiv \Theta\left(\alpha, \lambda_{m}(q+\varepsilon h)+q+\varepsilon h\right) \\
& =\Theta\left(\alpha, \lambda_{m}(q)+q+\varepsilon(\ell(h)+h)+o(\varepsilon)\right) .
\end{aligned}
$$

Differentiating this equality in $\varepsilon$ at $\varepsilon=0$, we get

$$
\begin{aligned}
0 & =\int_{0}^{1} \partial_{q} \Theta\left(\alpha, \lambda_{m}(q)+q\right)(s) \cdot(\ell(h)+h(s)) d s \\
& =\left(\int_{0}^{1}\left|\hat{E}_{m}(s ; q)\right|^{p} d s\right) \cdot \ell(h)+\int_{0}^{1}\left|\hat{E}_{m}(s ; q)\right|^{p} \cdot h(s) d s,
\end{aligned}
$$


where (3.10) is used. Thus

$$
\begin{aligned}
\ell(h) & =-\frac{\int_{0}^{1}\left|\hat{E}_{m}(s ; q)\right|^{p} \cdot h(s) d s}{\int_{0}^{1}\left|\hat{E}_{m}(s ; q)\right|^{p} d s} \\
& =-\int_{0}^{1} \frac{\left|\hat{E}_{m}(s ; q)\right|^{p}}{\left\|\hat{E}_{m}(\cdot ; q)\right\|_{p}^{p}} \cdot h(s) d s \\
& =\int_{0}^{1}\left(-\left|E_{m}(s ; q)\right|^{p}\right) \cdot h(s) d s .
\end{aligned}
$$

See definition (3.7) for $E_{m}(s ; q)$. Therefore we have $\partial_{q} \lambda_{m}(q)(t)=-\left|E_{m}(t ; q)\right|^{p}$. This proves (1.3) and (1.4).

Since the differentials (1.3) of the eigenvalues define negative linear functionals of $\left(\mathcal{L}^{\gamma},\|\cdot\|_{\gamma}\right)$, the eigenvalues possess the following monotonicity in potentials:

$$
\begin{aligned}
& q_{0}, q_{1} \in \mathcal{L}^{\gamma}, q_{1} \geq q_{0} \quad \Longrightarrow \quad \lambda_{m}\left(q_{1} ; \alpha, \beta\right) \leq \lambda_{m}\left(q_{0} ; \alpha, \beta\right), \\
& q_{0}, q_{1} \in \mathcal{L}^{\gamma}, q_{1} \succ q_{0} \quad \Longrightarrow \quad \lambda_{m}\left(q_{1} ; \alpha, \beta\right)<\lambda_{m}\left(q_{0} ; \alpha, \beta\right) .
\end{aligned}
$$

These can be proved using the monotonicity of $\Theta(\vartheta, q)$ in Lemma 2.7. For details, see 29].

3.4. Rotation numbers. Given $\gamma \in[1, \infty]$, the space $\mathcal{L}^{\gamma}=L^{\gamma}([0,1] ; \mathbb{R})$ can be identified as $L^{\gamma}(\mathbb{R} / \mathbb{Z} ; \mathbb{R})$ of 1 -periodic integrable functions. For any $q \in \mathcal{L}^{\gamma}$, the equation

$$
\left(\phi_{p}\left(x^{\prime}\right)\right)^{\prime}+q(t) \phi_{p}(x)=0, \quad t \in \mathbb{R},
$$

defines a dynamical system. In the $p$-polar coordinates, one has (2.2)-(2.3). Now the argument $\theta(t ; \vartheta, q)$ is well-defined for $t \in \mathbb{R}$. The evolution of $\theta(t ; \vartheta, q)$ defines the rotation number of equation (3.12), or of equation (2.3), by

$$
\varrho(q)=\varrho_{p}(q):=\lim _{t \rightarrow+\infty} \frac{\theta(t ; \vartheta, q)-\vartheta}{2 \pi_{p} t}=\lim _{t \rightarrow+\infty} \frac{\theta([t] ; \vartheta, q)-\vartheta}{2 \pi_{p}[t]} .
$$

It is independent of the initial value $\vartheta \in \mathbb{R}$. See [13, 17]. In the present case, $\varrho(q) \in[0, \infty)$. See [26, 29]. Due to the 1-periodicity of $q(t)$, we know that $\theta(t ; \vartheta, q)$ satisfies

$$
\theta(t+n ; \vartheta, q)=\theta(t ; \theta(n ; \vartheta, q), q), \quad \theta\left(t ; \vartheta+2 n \pi_{p}, q\right)=\theta(t ; \vartheta, q)+2 n \pi_{p}
$$

for any $n \in \mathbb{Z}$.

Theorem 3.5. As a functional, the rotation number

$$
\left(\mathcal{L}^{\gamma}, w_{\gamma}\right) \rightarrow \mathbb{R}, \quad q \rightarrow \varrho(q)
$$

is continuous in the weak topology of potentials.

Proof. Let us introduce

$\operatorname{Homeo}(\mathbb{R}):=\{h: \mathbb{R} \rightarrow \mathbb{R}: h$ is an orientation-preserving homeomorphism of $\mathbb{R}$ such that $h\left(\vartheta+2 n \pi_{p}\right) \equiv h(\vartheta)+2 n \pi_{p}$ for all $\vartheta \in \mathbb{R}$ and $\left.n \in \mathbb{Z}\right\}$.

The metric on Homeo $(\mathbb{R})$ is defined as

$$
d_{\infty}\left(h_{1}, h_{2}\right):=\max _{\vartheta \in \mathbb{R}}\left|h_{1}(\vartheta)-h_{2}(\vartheta)\right|=\max _{\vartheta \in\left[0,2 \pi_{p}\right]}\left|h_{1}(\vartheta)-h_{2}(\vartheta)\right| .
$$


It is known that for each $h \in \operatorname{Homeo}(\mathbb{R})$, the rotation number

$$
\hat{\varrho}(h):=\lim _{n \rightarrow+\infty} \frac{h^{n}(\vartheta)-\vartheta}{2 \pi_{p} n} \in \mathbb{R}
$$

is well-defined and independent of $\vartheta$. Moreover,

$$
\hat{\varrho}:\left(\operatorname{Homeo}(\mathbb{R}), d_{\infty}\right) \rightarrow \mathbb{R}
$$

is continuous 17, Proposition 11.1.6]. By Remark 2.4 for each $q \in \mathcal{L}^{\gamma}$, the solution $\theta(t ; \vartheta, q)$ defines a homeomorphism $\Theta(q)(\cdot):=\theta(1 ; \cdot, q) \in \operatorname{Homeo}(\mathbb{R})$. Due to the continuity of (2.12), one sees that

$$
\Theta:\left(\mathcal{L}^{\gamma}, w_{\gamma}\right) \rightarrow\left(\operatorname{Homeo}(\mathbb{R}), d_{\infty}\right), \quad q \rightarrow \Theta(q)
$$

is continuous. Now (3.13) shows that

$$
q \in\left(\mathcal{L}^{\gamma}, w_{\gamma}\right) \rightarrow \Theta(q) \in\left(\operatorname{Homeo}(\mathbb{R}), d_{\infty}\right) \rightarrow \varrho \hat{\varrho} \circ \Theta(q)=\varrho(q) \in \mathbb{R}
$$

is continuous.

For the case $p=2$, Theorem 3.5 was proved in 30 . For some continuity result of random dynamical systems on the circle, see [19.

3.5. Periodic and anti-periodic eigenvalues. Given $q \in \mathcal{L}^{\gamma}$. One can consider the periodic boundary condition

$$
x(1)-x(0)=x^{\prime}(1)-x^{\prime}(0)=0,
$$

and the anti-periodic boundary condition

$$
x(1)+x(0)=x^{\prime}(1)+x^{\prime}(0)=0 .
$$

In case $p \neq 2$, the structure of the eigenvalues of problem (1.1)-(3.14) and problem (1.1)-(3.15) is more complicated than the linear case $p=2$. See [5, 6]. It is known that for each $q \in \mathcal{L}^{\gamma}$, problems (1.1)-(3.14) and (1.1)-(3.15) have the so-called variational eigenvalues, denoted by

$$
\bar{\lambda}_{0}(q)<\underline{\lambda}_{1}(q) \leq \bar{\lambda}_{1}(q)<\cdots<\underline{\lambda}_{m}(q) \leq \bar{\lambda}_{m}(q)<\cdots
$$

such that $\underline{\lambda}_{m}(q)$ and $\bar{\lambda}_{m}(q)$ are the eigenvalues of (1.1)-(3.14) (of (1.1)-(3.15), respectively) when $m$ is even (odd, respectively). Notice that $\underline{\lambda}_{0}(q)$ is valid. In the linear case $p=2$, these have exhausted all periodic and anti-periodic eigenvalues for any potential $q(t)$. See 21]. The eigenvalues in (3.16) are also called the rotational eigenvalues in 29 , because they can be characterized using rotation numbers in the following way:

$$
\begin{aligned}
\bar{\lambda}_{0}(q) & =\max \{\lambda \in \mathbb{R}: \varrho(\lambda+q)=0\}, \\
\bar{\lambda}_{m}(q) & =\min \{\lambda \in \mathbb{R}: \varrho(\lambda+q)=m / 2\}, \\
\bar{\lambda}_{m}(q) & =\max \{\lambda \in \mathbb{R}: \varrho(\lambda+q)=m / 2\},
\end{aligned}
$$

where $m \in \mathbb{N}$. See [29, Theorem 3.3]. It is remarkable that there is some potential $q \in \mathrm{E}^{\infty}$ such that besides these eigenvalues in (3.16), problem (1.1)-(3.14) will admit some non-variational eigenvalues [5, 6. Some characterization different from (3.17)-(3.19) on those non-variational eigenvalues was given there. In this paper we are only interested in variational eigenvalues because they are easily handled and are more useful [1, 9, 14. 
Theorem 3.6. As functionals of potentials, variational eigenvalues are also continuous in weak topologies. That is, the following mappings are continuous:

$$
\left(\mathcal{L}^{\gamma}, w_{\gamma}\right) \rightarrow \mathbb{R}, \quad q \rightarrow \underline{\lambda}_{m}(q), \quad q \rightarrow \bar{\lambda}_{m}(q) .
$$

Proof. As in 30, due to the rotation number approach (3.17)-(3.19), we can introduce the functionals $\underline{\Theta}, \bar{\Theta}:\left(\mathcal{L}^{\gamma}, w_{\gamma}\right) \rightarrow \mathbb{R}$ by

$$
\underline{\Theta}(q):=\max _{\vartheta \in\left[0,2 \pi_{p}\right]}(\Theta(\vartheta, q)-\vartheta), \quad \bar{\Theta}(q):=\min _{\vartheta \in\left[0,2 \pi_{p}\right]}(\Theta(\vartheta, q)-\vartheta) .
$$

From the continuity of (2.12), one sees that both functionals $\underline{\Theta}$ and $\bar{\Theta}$ are continuous in $\left(\mathcal{L}^{\gamma}, w_{\gamma}\right)$. Now

$$
\begin{aligned}
& \lambda=\underline{\lambda}_{m}(q) \quad \Longleftrightarrow \quad \underline{\Theta}(\lambda+q)=m \pi_{p}, \\
& \lambda=\bar{\lambda}_{m}(q) \quad \Longleftrightarrow \quad \bar{\Theta}(\lambda+q)=m \pi_{p} .
\end{aligned}
$$

See [29, Theorem 3.3]. Equalities (3.21) and (3.22) are the equations for variational eigenvalues. One may compare them with equations (3.2) for separated eigenvalues. In the proof of the continuity of separated eigenvalues in weak topologies, the crucial fact we used was the monotonicity (3.5) of $\Theta(\alpha, \lambda+q)$ in $\lambda$. By Lemma 2.7, it is easy to see from (3.20) that both $\underline{\Theta}(\lambda+q)$ and $\bar{\Theta}(\lambda+q)$ have the monotonicity as (3.5). Now the continuity of $\underline{\lambda}_{m}(q)$ and $\bar{\lambda}_{m}(q)$ in $q \in\left(\mathcal{L}^{\gamma}, w_{\gamma}\right)$ can be obtained in a similar way.

Remark 3.7. Since $\bar{\lambda}_{0}(q)$ is a simple eigenvalue, the following mapping,

$$
\left(\mathcal{L}^{\gamma},\|\cdot\|_{\gamma}\right) \rightarrow \mathbb{R}, \quad q \rightarrow \bar{\lambda}_{0}(q),
$$

is continuously differentiable at all potentials $q$. Moreover, it can be proved that

$$
\partial_{q} \bar{\lambda}_{0}(q)=-\left|\bar{E}_{0}(\cdot ; q)\right|^{p},
$$

where $\bar{E}_{0}(t ; q)$ is a normalized eigenfunction associated with $\bar{\lambda}_{0}(q)$. For higherorder variational eigenvalues, due to the so-called coexistence of periodic and antiperiodic eigenvalues [7] or the so-called parametric resonance [3], $\underline{\lambda}_{m}(q)$ and $\bar{\lambda}_{m}(q)$, $m \in \mathbb{N}$, cannot be continuously differentiable in $q \in\left(\mathcal{L}^{\gamma},\|\cdot\|_{\gamma}\right)$ at all potentials. For example, $\underline{\lambda}_{m}(q)$ and $\bar{\lambda}_{m}(q), m \in \mathbb{N}$, are always not continuously differentiable at constant potentials. Similarly, the rotation number $\varrho(q)$ is also not continuously differentiable at some potentials such as $q=0$. See [30].

\section{EigenValues of the $p$-Laplacian With Weights}

4.1. Continuity of weighted eigenvalues in weak topologies. Given $\gamma \in$ $[1, \infty]$, the set $\mathcal{W}^{\gamma} \subset \mathcal{L}^{\gamma}$ of weights is defined by (1.9). Any $\rho \in \mathcal{W}^{\gamma}$ is called a (positive) definite weight.

Given a definite weight $\rho \in \mathcal{W}^{\gamma}$, we consider the eigenvalue problem

$$
\left(\phi_{p}\left(x^{\prime}\right)\right)^{\prime}+\mu \rho(t) \phi_{p}(x)=0,
$$

with the Dirichlet boundary condition (1.8). In the terminology of (3.1), condition (1.8) corresponds to $\alpha=\beta=-\pi_{p} / 2$. Since $\rho \succ 0$, the eigenvalue problem is clear. That is, the weighted eigenvalues of (4.1)-(1.8) are:

$$
(0<) \mu_{1}(\rho)<\mu_{2}(\rho)<\cdots<\mu_{m}(\rho)<\cdots, \quad \mu_{m}(\rho) \rightarrow+\infty .
$$

For detailed proofs, see, for example, $[15,28$. 
Since we are only considering the boundary condition (1.8), for $q \in \mathcal{L}^{\gamma}$, we make use of the following simple notation:

$$
\Theta(q):=\Theta\left(-\pi_{p} / 2, q\right)=\theta\left(1 ;-\pi_{p} / 2, q\right), \quad X(t ; q):=X\left(t ;-\pi_{p} / 2, q\right) .
$$

Now the eigenvalue $\mu=\mu_{m}(\rho), m \in \mathbb{N}$, is determined by the following equation:

$$
\Theta(\mu \rho)=-\pi_{p} / 2+m \pi_{p} .
$$

By the monotonicity of $\Theta(\cdot)$ of Lemma 2.7 one also has the following monotonicity results for weighted eigenvalues.

Lemma 4.1. Let $m \in \mathbb{N}$ be specified. Then

$$
\begin{aligned}
& \rho_{0}, \rho_{1} \in \mathcal{W}^{\gamma}, \rho_{1} \geq \rho_{0} \quad \Longrightarrow \quad \mu_{m}\left(\rho_{1}\right) \leq \mu_{m}\left(\rho_{0}\right), \\
& \rho_{0}, \rho_{1} \in \mathcal{W}^{\gamma}, \rho_{1} \succ \rho_{0} \quad \Longrightarrow \quad \mu_{m}\left(\rho_{1}\right)<\mu_{m}\left(\rho_{0}\right) .
\end{aligned}
$$

Notice that $\mathcal{W}^{\gamma} \subset \mathcal{L}^{\gamma}$. Besides the usual topology induced by $\|\cdot\|_{\gamma}$, one also has the weak topology $w_{\gamma}$ in the space $\mathcal{W}^{\gamma}$.

Theorem 4.2. Given $\gamma \in[1, \infty]$ and $m \in \mathbb{N}$, the functional

$$
\left(\mathcal{W}^{\gamma}, w_{\gamma}\right) \rightarrow(0, \infty), \quad \rho \rightarrow \mu_{m}(\rho)
$$

is continuous. That is, if $\rho_{n} \rightarrow \rho_{0}$ in $\left(\mathcal{W}^{\gamma}, w_{\gamma}\right)$, one has $\mu_{m}\left(\rho_{n}\right) \rightarrow \mu_{m}\left(\rho_{0}\right)$.

Proof. The proof is similar to that of Theorem 1.1. Suppose that $\rho_{n} \rightarrow \rho_{0}$ in $\left(\mathcal{W}^{\gamma}, w_{\gamma}\right)$. Denote $\nu_{n}:=\mu_{m}\left(\rho_{n}\right), n \in \mathbb{Z}^{+}$. One has

$$
\Theta\left(\nu_{n} \rho_{n}\right)=-\pi_{p} / 2+m \pi_{p}, \quad n \in \mathbb{Z}^{+} .
$$

We aim to prove that $\nu_{n} \rightarrow \nu_{0}$ as $n \rightarrow \infty$. Notice that $\nu_{0}>0$. If $\nu_{n} \nrightarrow \nu_{0}$, passing to a subsequence, we assume that there exists $\varepsilon_{0} \in\left(0, \nu_{0}\right)$ such that

$$
\left|\nu_{n}-\nu_{0}\right| \geq \varepsilon_{0} \quad \forall n \geq 1 \text {. }
$$

Without loss of generality, we may assume further that

$$
0<\nu_{n} \leq \nu_{0}-\varepsilon_{0} \quad \forall n \geq 1 .
$$

Thus

$$
\rho_{n} \succ 0 \Longrightarrow \nu_{n} \rho_{n} \leq\left(\nu_{0}-\varepsilon_{0}\right) \rho_{n} .
$$

By Lemma 2.7 and equalities (4.3), we have

$$
-\pi_{p} / 2+m \pi_{p}=\Theta\left(\nu_{n} \rho_{n}\right) \leq \Theta\left(\left(\nu_{0}-\varepsilon_{0}\right) \rho_{n}\right) \quad \forall n \geq 1 .
$$

Letting $n \rightarrow \infty$ and noticing that $\left(\nu_{0}-\varepsilon_{0}\right) \rho_{n} \rightarrow\left(\nu_{0}-\varepsilon_{0}\right) \rho_{0}$ in $\left(\mathcal{L}^{\gamma}, w_{\gamma}\right)$, we know from Theorem 2.3 and Lemma 2.7 that

$$
-\pi_{p} / 2+m \pi_{p} \leq \Theta\left(\left(\nu_{0}-\varepsilon_{0}\right) \rho_{0}\right)<\Theta\left(\nu_{0} \rho_{0}\right),
$$

because $\rho_{0} \succ 0$ and $\left(\nu_{0}-\varepsilon_{0}\right) \rho_{0} \prec \nu_{0} \rho_{0}$. However, this contradicts the equality for $\nu_{0}$. See (4.3) with $n=0$. 
4.2. Continuously differentiable extension of weighted eigenvalues. The space $\left(\mathcal{L}^{\gamma},\|\cdot\|_{\gamma}\right)$ for potentials is a Banach space. However, the space $\mathcal{W}^{\gamma}$ for positive-definite weights is only a cone of $\left(\mathcal{L}^{\gamma},\|\cdot\|_{\gamma}\right)$. In case $\gamma \in[1, \infty)$, due to the restriction $\rho \succ 0, \mathcal{W}^{\gamma}$ does not have any interior point in the space $\left(\mathcal{L}^{\gamma},\|\cdot\|_{\gamma}\right)$. To make sense of the differentials of $\mu_{m}(\rho)$ in $\rho$, we must extend functionals $\mu_{m}(\rho)$, $\rho \in \mathcal{W}^{\gamma}$, to some open subset of $\left(\mathcal{L}^{\gamma},\|\cdot\|_{\gamma}\right)$. This will lead to weighted eigenvalues of indefinite weights [2, 8, 11.

Now let $\omega \in \mathcal{L}^{\gamma} \backslash\{0\}$, which may be sign-changing. Consider the weighted eigenvalue problem (1.7)-(1.8). In terms of the argument $\Theta(\cdot), \mu \in \mathbb{R}$ is an eigenvalue of (1.7) - (1.8) if and only if $\mu$ satisfies

$$
\Theta(\mu \omega)=-\pi_{p} / 2+m \pi_{p}
$$

for some $m \in \mathbb{N}$. Notice that

$$
-\pi_{p} / 2<\Theta(0)<0
$$

Hence all eigenvalues of (1.7)-(1.8) are necessarily non-zero. Though it is a classical problem, different from the eigenvalue theory for the $p$-Laplacian with potentials, it seems that the eigenvalue theory for (1.7)-(1.8) has not been completely established for general integrable sign-changing weights from $\mathrm{L}^{\gamma}, \gamma \in[1, \infty)$.

In order to state some known results, let us introduce the following notation. For any $\omega \in \mathcal{L}^{\gamma}$, we write

$$
\omega_{+}(t):=\max (\omega(t), 0), \quad \omega_{-}(t)=\max (-\omega(t), 0) .
$$

Hence $\omega(t) \equiv \omega_{+}(t)-\omega_{-}(t)$. Denote

$$
\mathcal{W}_{ \pm}^{\gamma}:=\left\{\omega \in \mathcal{L}^{\gamma}: \omega_{ \pm} \succ 0\right\}=\left\{\omega \in \mathcal{L}^{\gamma}: \int_{0}^{1} \omega_{ \pm}(t) d t>0\right\}, \quad \gamma \in[1, \infty] .
$$

In case $\gamma=\infty$ and $\omega \in \mathcal{W}_{+}^{\infty}$, the authors of [2] have applied the minimax technique to prove that all positive eigenvalues of (1.7)-(1.8) are given by

$$
(0<) \mu_{1}^{+}(\omega)<\mu_{2}^{+}(\omega)<\cdots<\mu_{m}^{+}(\omega)<\cdots, \quad \mu_{m}^{+}(\omega) \rightarrow+\infty .
$$

It is not difficult to see that $\mu=\mu_{m}^{+}(\omega) \in(0, \infty)$ satisfies (4.5). For piecewise continuous weights which are necessarily in $\mathrm{E}^{\infty}$, an ODE approach is given in [11. See also [8] for the study of the (positive) principal eigenvalues $\mu_{1}^{+}(\omega)$. Dually, for $\omega \in \mathcal{W}_{-}^{\infty}$, problem (1.7)-(1.8) has a sequence of negative eigenvalues

$$
\mu_{m}^{-}(\omega) \equiv-\mu_{m}^{+}(-\omega), \quad m \in \mathbb{N} .
$$

Consequently, for $\omega \in \mathcal{W}_{+}^{\infty} \cap \mathcal{W}_{-}^{\infty}$, called a sign-changing weight, one has eigenvalues $\left\{\mu_{m}^{ \pm}(\omega)\right\}_{m \in \mathbb{N}}$.

However, in case $\gamma \in[1, \infty)$, for those sign-changing weights $\omega \in \mathcal{W}_{+}^{\gamma} \cap \mathcal{W}_{-}^{\gamma}$, we are not able to find general references on problems (1.7)-(1.8), though the desired results can be expected. Considering this, we need to write the results of Theorem 4.3 in a moderate way. These will be used in the study of extremal problems in the next section.

Notice that $\mathcal{W}^{\gamma} \subset \mathcal{W}_{+}^{\gamma}$ and $\mathcal{W}_{+}^{\gamma} \subset\left(\mathcal{L}^{\gamma},\|\cdot\|_{\gamma}\right)$ is an open subset. Recall that, for any $m \in \mathbb{N}$ fixed, the following functional is well-defined:

$$
\mathcal{W}^{\gamma} \rightarrow(0, \infty), \quad \rho \rightarrow \mu_{m}(\rho) .
$$

We have the following local extension of the functional $\mu_{m}(\cdot)$. 
Theorem 4.3. Given $\gamma \in[1, \infty]$ and $m \in \mathbb{N}$, then, for any $\rho \in \mathcal{W}^{\gamma}$, there exists $\delta=\delta_{\rho, \gamma, m}>0$ such that

$$
B_{\delta}(\rho):=\left\{\omega \in \mathcal{L}^{\gamma}:\|\omega-\rho\|_{\gamma}<\delta\right\} \subset \mathcal{W}_{+}^{\gamma},
$$

and there exists a functional

$$
B_{\delta}(\rho) \rightarrow(0, \infty), \quad \omega \rightarrow \mu_{m}^{+}(\omega),
$$

such that $\mu=\mu_{m}^{+}(\omega) \in(0, \infty)$ satisfies (4.5) and

$$
\mu_{m}^{+}(w)=\mu_{m}(w), \quad w \in B_{\delta}(\rho) \cap \mathcal{W}^{\gamma} .
$$

Moreover, $\mu_{m}^{+}(\omega)$ is continuously differentiable in $\omega \in\left(B_{\delta}(\rho),\|\cdot\|_{\gamma}\right)$, with the differential

$$
\partial_{\omega} \mu_{m}^{+}(\omega)(t)=-\mu_{m}^{+}(\omega) \cdot\left|E_{m}^{+}(t ; \omega)\right|^{p} \in \mathcal{C}^{0} \subset\left(\mathcal{L}^{\gamma},\|\cdot\|_{\gamma}\right)^{*},
$$

where $E_{m}^{+}(t ; \omega)$ is an eigenfunction associated with $\mu_{m}^{+}(\omega)$ and satisfies the following normalization condition:

$$
\int_{0}^{1}\left|E_{m}^{+}(t ; \omega)\right|^{p} \cdot \omega(t) d t=1
$$

Proof. Since $\mu_{m}^{+}(\omega) \in(0, \infty)$ will be constructed from equation (4.5), it is necessarily a weighted eigenvalue of (1.7)-(1.8).

Step 1. The existence of $B_{\delta}(\rho)$ and $\mu_{m}^{+}(\cdot)$ will be proved by applying the Implicit Function Theorem to equation (4.5). By Theorem 2.5, $\Theta(\mu \omega)$ is continuously differentiable in $(\mu, \omega) \in \mathbb{R} \times\left(\mathcal{L}^{\gamma},\|\cdot\|_{\gamma}\right)$. Moreover, $(\mu, \omega)=\left(\mu_{m}(\rho), \rho\right)$ satisfies (4.5). In order to apply the Implicit Function Theorem, we need only verify the following condition:

$$
\left.\frac{d}{d \mu} \Theta(\mu \rho)\right|_{\mu=\mu_{m}(\rho)} \neq 0 .
$$

More generally, let $\omega \in \mathcal{L}^{\gamma}$ be fixed. The derivative of $\Theta(\mu \omega)$ with respect to $\mu$ is

$$
\frac{d}{d \mu} \Theta(\mu \omega)=\left.\partial_{q} \Theta(q)\right|_{q=\mu \omega} \cdot \omega=\int_{0}^{1}|X(t ; \mu \omega)|^{p} \cdot \omega(t) d t .
$$

See (2.15) and (2.20). Recall that $X(t ; \mu \omega)$ satisfies $X(0 ; \mu \omega)=0$ and

$$
\left(\phi_{p}\left(X^{\prime}(t ; \mu \omega)\right)\right)^{\prime}+\mu \omega(t) \phi_{p}(X(t ; \mu \omega))=0 .
$$

Multiplying by $X(t ; \mu \omega)$ and integrating over $[0,1]$, we can get

$$
\begin{aligned}
& \mu \cdot \int_{0}^{1}|X(t ; \mu \omega)|^{p} \cdot \omega(t) d t \\
= & -\int_{0}^{1} X(t ; \mu \omega) \cdot\left(\phi_{p}\left(X^{\prime}(t ; \mu \omega)\right)\right)^{\prime} d t \\
= & \int_{0}^{1}\left|X^{\prime}(t ; \mu \omega)\right|^{p} d t-\left.X(t ; \mu \omega) \phi_{p}\left(X^{\prime}(t ; \mu \omega)\right)\right|_{t=0} ^{1} \\
= & \int_{0}^{1}\left|X^{\prime}(t ; \mu \omega)\right|^{p} d t-X(1 ; \mu \omega) \phi_{p}\left(X^{\prime}(1 ; \mu \omega)\right)
\end{aligned}
$$

because $X(0 ; \mu \omega)=0$. Substituting into (4.11), we get another equality:

$$
\frac{d}{d \mu} \Theta(\mu \omega)=\frac{\int_{0}^{1}\left|X^{\prime}(t ; \mu \omega)\right|^{p} d t-X(1 ; \mu \omega) \phi_{p}\left(X^{\prime}(1 ; \mu \omega)\right)}{\mu}
$$


for $\mu \neq 0$. Notice that for general $\mu$, we have no further information on $X(1 ; \mu \omega)$ and $X^{\prime}(1 ; \mu \omega)$.

However, if $\mu \in(0, \infty)$ satisfies (4.5), we know that $\mu$ is necessarily a positive eigenvalue of (1.7)-(1.8) and $X(1 ; \mu \omega)=0$. Now (4.13) shows that

$$
\left.\frac{d}{d \mu} \Theta(\mu \omega)\right|_{\mu>0 \text { satisfies (4.5) }}=\frac{\int_{0}^{1}\left|X^{\prime}(t ; \mu \omega)\right|^{p} d t}{\mu}>0 .
$$

Notice that $(\mu, \omega)=\left(\mu_{m}(\rho), \rho\right)$ satisfies (4.5). Consequently, we know that the derivative (4.10) is actually positive.

Now the Implicit Function Theorem shows that there exists $\delta=\delta_{\rho, \gamma, m}>0$ and a continuously differentiable function $\mu_{m}^{+}:\left(B_{\delta}(\rho),\|\cdot\|_{\gamma}\right) \rightarrow(0, \infty)$ such that $B_{\delta}(\rho) \subset \mathcal{W}_{+}^{\gamma}$ and $\mu=\mu_{m}^{+}(\omega)$ satisfies (4.5) for all $\omega \in B_{\delta}(\rho)$. Due to equations (4.2) and (4.5), we know that $\mu_{m}^{+}(\cdot)$ is a local extension of $\mu_{m}(\cdot)$, i.e., (4.7) is satisfied.

Step 2. For any $\omega \in B_{\delta}(\rho)$, as mentioned before, $\mu_{m}^{+}(\omega)$ is a positive eigenvalue of (1.7)-(1.8). One can introduce the corresponding eigenfunction

$$
\tilde{E}_{m}(t ; \omega):=X\left(t ; \mu_{m}^{+}(\omega) \omega\right) .
$$

Let us now evaluate the differential of $\mu_{m}^{+}(\omega)$ at $\omega \in B_{\delta}(\rho)$. To this end, let $h \in \mathcal{L}^{\gamma}$ and $\varepsilon \in \mathbb{R}$ with $|\varepsilon|$ small. Write

$$
\mu_{m}^{+}(\omega+\varepsilon h)=\mu_{m}^{+}(\omega)+\varepsilon \ell(h)+o(\varepsilon),
$$

where $\ell(h) \in \mathbb{R}$ is linear in $h$. Now we get from (4.5) that

$$
\begin{aligned}
-\pi_{p} / 2+m \pi_{p} & =\Theta\left(\mu_{m}^{+}(\omega+\varepsilon h)(\omega+\varepsilon h)\right) \\
& =\Theta\left(\left(\mu_{m}^{+}(\omega)+\varepsilon \ell(h)+o(\varepsilon)\right)(\omega+\varepsilon h)\right) \\
& =\Theta\left(\mu_{m}^{+}(\omega) \omega+\varepsilon\left(\ell(h) \omega+\mu_{m}^{+}(\omega) h\right)+o(\varepsilon)\right) .
\end{aligned}
$$

Differentiating this with respect to $\varepsilon$ at $\varepsilon=0$, using the eigenfunction $\tilde{E}_{m}(t ; \omega)$, we can apply (2.15) and (2.20) to obtain

$$
\begin{aligned}
0 & =\int_{0}^{1}\left|\tilde{E}_{m}(t ; \omega)\right|^{p} \cdot\left(\ell(h) \omega(t)+\mu_{m}^{+}(\omega) h(t)\right) d t \\
& =\left(\int_{0}^{1}\left|\tilde{E}_{m}(t ; \omega)\right|^{p} \cdot \omega(t) d t\right) \cdot \ell(h)+\mu_{m}^{+}(\omega) \int_{0}^{1}\left|\tilde{E}_{m}(t ; \omega)\right|^{p} \cdot h(t) d t .
\end{aligned}
$$

Thus

$$
\partial_{\omega} \mu_{m}^{+}(\omega) \cdot h=\ell(h)=-\mu_{m}^{+}(\omega) \cdot \int_{0}^{1} \frac{\left|\tilde{E}_{m}(t ; \omega)\right|^{p}}{\int_{0}^{1}\left|\tilde{E}_{m}(s ; \omega)\right|^{p} \cdot \omega(s) d s} \cdot h(t) d t .
$$

Let $\mu=\mu_{m}^{+}(\omega)>0$ in (4.12). We have

$$
A:=\int_{0}^{1}\left|\tilde{E}_{m}(s ; \omega)\right|^{p} \cdot \omega(s) d s=\frac{1}{\mu_{m}^{+}(\omega)} \int_{0}^{1}\left|\tilde{E}_{m}^{\prime}(s ; \omega)\right|^{p} d s>0 .
$$

We can define an eigenfunction associated with $\mu_{m}^{+}(\omega)$ by

$$
E_{m}^{+}(t ; \omega):=A^{-1 / p} \cdot \tilde{E}_{m}(t ; \omega) .
$$

Now (4.8) can be obtained from (4.14). Moreover, by the definition of $E_{m}^{+}(t ; \omega)$, it satisfies the normalization condition (4.9).

For any $\omega \in B_{\delta}(\rho)$, formula (4.8) shows that $\partial_{\omega} \mu_{m}^{+}(\omega)(t)$ is negative. Since $B_{\delta}(\rho)$ is convex, one has the following monotonicity for $\mu_{m}^{+}(\omega)$. 
Lemma 4.4. Let $\mu_{m}^{+}: B_{\delta}(\rho) \rightarrow(0, \infty)$ be as in Theorem 4.3. Then

$$
\begin{array}{lll}
\omega_{0}, \omega_{1} \in B_{\delta}(\rho), \omega_{1} \geq \omega_{0} & \Longrightarrow & \mu_{m}^{+}\left(\omega_{1}\right) \leq \mu_{m}^{+}\left(\omega_{0}\right), \\
\omega_{0}, \omega_{1} \in B_{\delta}(\rho), \omega_{1} \succ \omega_{0} & \Longrightarrow & \mu_{m}^{+}\left(\omega_{1}\right)<\mu_{m}^{+}\left(\omega_{0}\right) .
\end{array}
$$

For $L^{\infty}$ weights $\omega$, global monotonicity results for $\mu_{m}^{+}(\omega)$ have been proved in [2, 8) using the minimax characterization. However, the proof of our local results of Lemma 4.4 is based on the differentials.

Remark 4.5. (i) We have not yet known whether $\mu_{m}^{+}: B_{\delta}(\rho) \rightarrow(0, \infty)$ is also continuous in $w_{\gamma}$. The reasons are as follows. For a sign-changing weight $\omega \in B_{\delta}(\rho)$, the function

$$
(0, \infty) \rightarrow \mathbb{R}, \quad \mu \rightarrow \Theta(\mu \omega),
$$

is, in general, not increasing. Hence the inequalities such as (3.4) and (4.4) are now incorrect. Thus the proofs of Theorem 1.1 and Theorem 4.2 do not work in the present case.

(ii) In Theorem 4.3, we have found a local continuation $\mu_{m}^{+}(\omega)$ of the weighted eigenvalue $\mu_{m}(\rho)$ which gives positive eigenvalues of (1.7)-(1.8) for some signchanging weights in $\mathcal{L}^{\gamma}, \gamma \in[1, \infty)$. However, Theorem 4.3 has not given a general spectrum result for sign-changing integrable weights, even for those weights which are close to $\mathcal{W}^{\gamma}$. The reason is that the intersection

$$
\bigcap_{m \in \mathbb{N}} B_{\delta_{\rho, \gamma, m}}(\rho)
$$

may shrink to a single point $\rho$.

(iii) Once the spectrum of (1.7)-(1.8) can be established for all integrable weights $\omega \in \mathcal{W}_{+}^{\gamma}, \gamma \in[1, \infty)$, the eigenvalues $\mu_{m}^{+}(\omega)$ are globally continuously differentiable. Moreover, results (4.8) and (4.9) hold for all $\omega \in \mathcal{W}_{+}^{\gamma}$. Dually, similar results can be established for $\mu_{m}^{-}(\omega), \omega \in \mathcal{W}_{-}^{\gamma}$.

\section{Applications to extremal problems of eigenvalues}

In this section, we will present some applications. Further work will be undertaken in other papers.

5.1. The extremal problem of Krein. The following is a classical extremal problem, which was solved by Krein [18] for the Laplacian case and by Yan and Zhang 25] for the $p$-Laplacian case.

Let $0<r \leq h<\infty$ be fixed. Define the set

$$
E_{r, h}:=\left\{\rho \in \mathrm{E}^{1}: 0 \leq \rho(t) \leq h \text { a.e. } t \in[0,1], \int_{0}^{1} \rho(t) d t=r\right\} \subset \mathcal{W}_{+}^{1} .
$$

Given an exponent $p \in(1, \infty)$, for any $\rho \in E_{r, h}$, we use $\mu_{m}(\rho), m \in \mathbb{N}$, to denote the weighted Dirichlet eigenvalues of (4.1). Notice that they depend on $p$ as well. The following extremal problems,

$$
\mathbf{L}_{m}(r, h):=\inf _{\rho \in E_{r, h}} \mu_{m}(\rho), \quad \mathbf{M}_{m}(r, h):=\sup _{\rho \in E_{r, h}} \mu_{m}(\rho),
$$

have been solved in [18, 25], where there are two crucial steps.

The first step is to prove that both extremal values of (5.2) can be attained by some weights in $E_{r, h}$. This step is now quite easy by the weak continuity of 
Theorem 4.2 because the set $E_{r, h}$ of (5.1) is a sequentially compact subset of $\mathrm{E}^{1}$ with respect to the weak topology $w_{1}$. In fact,

$$
\left\{\rho \in \mathrm{L}^{1}: 0 \leq \rho \leq h\right\}
$$

is an order interval and is sequentially compact in $\left(\mathrm{E}^{1}, w_{1}\right)$, and

$$
\left\{\rho \in \mathrm{E}^{1}: \int_{0}^{1} \rho(t) d t=r\right\}
$$

is a sequentially closed subset of $\left(\mathrm{E}^{1}, w_{1}\right)$. Notice that the restriction $h$ on the heights of weights is necessary to ensure that $E_{r, h}$ is sequentially compact in $\left(\mathrm{E}^{1}, w_{1}\right)$.

The second step is to find the minimizers and maximizers. This step is very tricky, depending on the problem studied. For the $L^{1}$ potentials and weights, the solution is suggested by Pontrjagin's Maximum Principle [27, §§48.6-48.8].

The explicit expressions of $\mathbf{L}_{m}(r, h)$ and $\mathbf{M}_{m}(r, h)$ can be found in [25]. That is,

$$
\mathbf{L}_{m}(r, h)=\frac{m^{p} 2^{p} h^{p-1}}{r^{p}} \chi_{p}\left(\frac{r}{h}\right), \quad \mathbf{M}_{m}(r, h)=\frac{m^{p} \pi_{p}^{p} h^{p-1}}{r^{p}},
$$

where, for any $t \in(0,1), \chi=\chi_{p}(t) \in\left(0,\left(-\pi_{p} / 2\right)^{p}\right)$ is the least positive root of the following equation:

$$
\chi^{1 / p} \frac{\left(S_{p}\left(\chi^{1 / p}\right)\right)^{p^{*}-1}}{C_{p}\left(\chi^{1 / p}\right)}=\frac{t}{1-t},
$$

and, for $t=1$,

$$
\chi_{p}(1):=\lim _{t \rightarrow 1^{-}} \chi_{p}(t)=\left(\pi_{p} / 2\right)^{p} .
$$

In [16], Karaa has studied several extremal problems concerned with eigenvalues or weighted eigenvalues. One step is also the existence of minimizers or maximizers. This can also be explained using the continuity of these eigenvalues in weak topologies.

5.2. The extremal problem in balls. Let $\gamma \in[1, \infty]$. For any $r \in(0, \infty)$, we can define the ball

$$
B_{\gamma}[r]:=\left\{q \in \mathcal{L}^{\gamma}:\|q\|_{\gamma} \leq r\right\} .
$$

The extremal problems for eigenvalues of the $p$-Laplacian with potentials in $B_{\gamma}[r]$,

$$
\mathbf{L}_{m, \gamma}^{p}(r):=\inf \left\{\lambda_{m}(q): q \in B_{\gamma}[r]\right\}, \quad \mathbf{M}_{m, \gamma}^{p}(r):=\sup \left\{\lambda_{m}(q): q \in B_{\gamma}[r]\right\},
$$

where $m \in \mathbb{N}$, are of interest. In case $\gamma \in(1, \infty]$, it is well known that $B_{\gamma}[r]$ is a sequentially compact subset of $\left(\mathcal{L}^{\gamma}, w_{\gamma}\right)$. An immediate result of Theorem 1.1 is

Corollary 5.1. Let $\gamma \in(1, \infty]$. Then, for any $r \in[0, \infty)$, the extremal values $\mathbf{L}_{m, \gamma}^{p}(r)$ and $\mathbf{M}_{m, \gamma}^{p}(r)$ of (5.5) are finite.

For the most interesting case $\gamma=1$, the finiteness of $\mathbf{L}_{m, 1}^{p}(r)$ and $\mathbf{M}_{m, 1}^{p}(r)$ does not follow from Theorem 1.1 in a direct way. By some topological fact on $L^{\gamma}$ balls in [31, one has the following limiting equalities:

$$
\mathbf{L}_{m, 1}^{p}(r)=\lim _{\gamma \downarrow 1} \mathbf{L}_{m, \gamma}^{p}(r), \quad \mathbf{M}_{m, 1}^{p}(r)=\lim _{\gamma \downarrow 1} \mathbf{M}_{m, \gamma}^{p}(r) .
$$

In another paper, by examining $\mathbf{L}_{m, \gamma}^{p}(r)$ and $\mathbf{M}_{m, \gamma}^{p}(r), \gamma \in(1, \infty)$, in a careful way, we will use (5.6) to prove that both $\mathbf{L}_{m, 1}^{p}(r)$ and $\mathbf{M}_{m, 1}^{p}(r)$ are also finite for all $r$. 
In fact, these two important extremal values can be evaluated explicitly by using $r$, as done in 31] for the case $p=2$.

Due to the continuity result of Theorem 3.5. one can study the following extremal problem for the rotation number:

$$
\mathbf{R}_{\gamma}^{p}(r):=\sup \left\{\varrho(\rho): \rho \in B_{\gamma}[r]\right\} .
$$

Like the consideration in 12 for the case $p=2$, we can obtain the following upper bound:

$$
\mathbf{R}_{\gamma}^{p}(r) \leq C(\gamma, p) r^{1 / p}, \quad r \in[0, \infty), \gamma \in[1, \infty] .
$$

Here $C(\gamma, p)$ is some constant which can be evaluated using the Sobolev constant $\mathbf{K}\left(p \gamma^{*}, p\right)$ of (5.19) below. However, problem (5.7) remans open.

5.3. An extremal problem on non-compact sets. Notice that the problems above are all defined in sets which are sequentially compact in weak topologies. In this subsection, we will give a complete solution to an extremal problem on a non-compact set of weights. Of particular interest is that we will use some methods which are completely different from those in [16, 18, 25].

Given $\gamma \in[1, \infty]$ and $r \in(0, \infty)$, one can define the positive semi-sphere

$$
E_{r}^{\gamma}:=\left\{\rho \in \mathcal{L}^{\gamma}: 0 \leq \rho(t) \text { a.e. } t \in[0,1],\|\rho\|_{\gamma}=r\right\} \subset \mathcal{W}^{\gamma} .
$$

Different from the previous two problems, the set $E_{r}^{\gamma}$ of weights is not sequentially compact even in the weak topology $w_{\gamma}$.

Let $p \in(1, \infty)$ be given. We use $\mu_{m}(\rho), m \in \mathbb{N}$, to denote weighted Dirichlet eigenvalues of (4.1) with $\rho \in \mathcal{W}^{\gamma}$. We are interested in the following extremal value:

$$
\mathbf{L}_{m, \gamma}(r):=\inf _{\rho \in E_{r}^{\gamma}} \mu_{m}(\rho),
$$

which is well-defined and is positive 28. Of particular interest is the case $\gamma=1$. In Theorem 5.6 below, we will give an answer to $\mathbf{L}_{m, \gamma}(r)$.

Preliminarily, as $\mu_{m}(c \rho)=\mu_{m}(\rho) / c$ for all $\rho \in \mathcal{W}^{\gamma}$ and $c>0$, by definition (5.8) of $\mathbf{L}_{m, \gamma}(r)$, it is easy to see that $\mathbf{L}_{m, \gamma}(r)$ satisfies

$$
0<r_{1}<r_{2}<\infty \Longrightarrow \frac{\mathbf{L}_{m, \gamma}\left(r_{2}\right)}{\mathbf{L}_{m, \gamma}\left(r_{1}\right)}=\frac{r_{1}}{r_{2}}<1
$$

In particular, we have the following result.

Lemma 5.2. Given $\gamma \in[1, \infty]$ and $m \in \mathbb{N}$, as a function of $r, \mathbf{L}_{m, \gamma}(\cdot):(0, \infty) \rightarrow$ $(0, \infty)$ is strictly decreasing.

We distinguish three cases for $\gamma \in[1, \infty]$.

Case $\gamma=\infty$. By the monotonicity results of Lemma 4.1 it is easy to see that

$$
\mathbf{L}_{m, \infty}(r)=\mu_{m}(r)=\frac{\left(m \pi_{p}\right)^{p}}{r} .
$$

Case $\gamma \in(1, \infty)$. The values $\mathbf{L}_{m, \gamma}(r)$ can be evaluated using the critical equations.

As $E_{r}^{\gamma}$ is not sequentially compact in $w_{\gamma}$, the existence of minimizers of (5.8) cannot be obtained directly from the continuity in the weak topology.

Lemma 5.3. For each $r \in(0, \infty)$, there exists some $\rho_{0}=\rho_{0}(\gamma, m, r, p) \in E_{r}^{\gamma}$ such that $\mu_{m}\left(\rho_{0}\right)=\mathbf{L}_{m, \gamma}(r)$. That is, the minimum of (5.8) can be attained. 
Proof. By definition of $\mathbf{L}_{m, \gamma}(r)$, there exists a sequence $\left\{\rho_{n}\right\}_{n \in \mathbb{N}}$,

$$
\rho_{n} \in E_{r}^{\gamma} \subset K_{r}^{\gamma}:=\left\{\rho \in \mathcal{L}^{\gamma}: \rho \geq 0,\|\rho\|_{\gamma} \leq r\right\}
$$

such that

$$
\lim _{n \rightarrow \infty} \mu_{m}\left(\rho_{n}\right)=\mathbf{L}_{m, \gamma}(r) .
$$

Notice that $K_{r}^{\gamma} \subset\left(\mathcal{L}^{\gamma},\|\cdot\|_{\gamma}\right)$ is a bounded closed convex set. Thus $K_{r}^{\gamma} \subset\left(\mathcal{L}^{\gamma}, w_{\gamma}\right)$ is sequentially compact. Without loss of generality, we may assume that

$$
\rho_{n} \rightarrow \rho_{0} \in K_{r}^{\gamma} \quad \text { in } w_{\gamma} .
$$

Hence $\rho_{0} \geq 0$ and $\left\|\rho_{0}\right\|_{\gamma} \leq r$.

Let us prove that $\rho_{0}$ is actually in $E_{r}^{\gamma}$. By (5.9) and (5.10), we have

$$
\mu_{m}\left(\rho_{n}\right) \rho_{n} \rightarrow \mathbf{L}_{m, \gamma}(r) \rho_{0} . \quad \text { in } w_{\gamma} .
$$

By Theorem 2.3, we get from

$$
\Theta\left(\mu_{m}\left(\rho_{n}\right) \rho_{n}\right)=-\pi_{p} / 2+m \pi_{p}
$$

the limiting equality

$$
\Theta\left(\mathbf{L}_{m, \gamma}(r) \rho_{0}\right)=-\pi_{p} / 2+m \pi_{p} .
$$

This shows that $\rho_{0} \neq 0$ because of (4.6). Thus $\rho_{0} \succ 0$ and $\rho_{0} \in \mathcal{W}^{\gamma}$. Now we can apply Theorem 4.2 to $\rho_{n}$ and get from (5.9),

$$
\mu_{m}\left(\rho_{0}\right)=\mathbf{L}_{m, \gamma}(r) .
$$

We assert that $\left\|\rho_{0}\right\|_{\gamma}=r$ and consequently $\rho_{0} \in E_{r}^{\gamma}$. Otherwise, $\hat{r}:=\left\|\rho_{0}\right\|_{\gamma} \in(0, r)$. In this case, (5.11) implies that

$$
\mathbf{L}_{m, \gamma}(\hat{r}) \leq \mu_{m}\left(\rho_{0}\right)=\mathbf{L}_{m, \gamma}(r),
$$

which contradicts Lemma 5.2

Lemma 5.4. Given $r \in(0, \infty)$ and $m \in \mathbb{N}$, as a function of $\gamma, \mathbf{L}_{m, \cdot}(r):[1, \infty) \rightarrow$ $(0, \infty)$ is increasing.

Proof. Given $1 \leq \gamma_{1}<\gamma_{2}<\infty$, let $\rho_{0}:=\rho_{0}\left(\gamma_{2}, m, r, p\right)$ be the minimizer in Lemma 5.3. By the definition of $\mathbf{L}_{m, \gamma}(\cdot)$, one has

$$
\mathbf{L}_{m, \gamma_{1}}\left(\left\|\rho_{0}\right\|_{\gamma_{1}}\right) \leq \mu_{m}\left(\rho_{0}\right)=\mathbf{L}_{m, \gamma_{2}}(r) .
$$

The Hölder inequality implies that $\left\|\rho_{0}\right\|_{\gamma_{1}} \leq\left\|\rho_{0}\right\|_{\gamma_{2}}=r$. Therefore it follows from Lemma 5.2 that $\mathbf{L}_{m, \gamma_{1}}(r) \leq \mathbf{L}_{m, \gamma_{1}}\left(\left\|\rho_{0}\right\|_{\gamma_{1}}\right) \leq \mathbf{L}_{m, \gamma_{2}}(r)$, proving the lemma.

Due to the restriction $\rho \succ 0$ in $E_{r}^{\gamma}$, the set $E_{r}^{\gamma}$ has boundary in the $\|\cdot\|_{\gamma}$ topology. It is not easy to apply the variational method to (5.8) to find the minimizers $\rho_{0}$ in a direct way. To overcome this, let us extend (5.8) to a minimum problem of a smooth functional under some smooth constraint.

Lemma 5.5. Let $\rho_{0} \in E_{r}^{\gamma}$ be as in Lemma 5.3 and $\delta=\delta_{\rho_{0}, m, \gamma}>0$ be as in Theorem 4.3. Denote

$$
\begin{aligned}
& B_{\delta}:=\left\{\omega \in \mathcal{L}^{\gamma}:\left\|\omega-\rho_{0}\right\|_{\gamma}<\delta\right\}, \quad S_{r}:=\left\{\omega \in \mathcal{L}^{\gamma}:\|\omega\|_{\gamma}=r\right\}, \\
& M_{\delta, r}:=B_{\delta} \cap S_{r} .
\end{aligned}
$$

Then we have

$$
\inf \left\{\mu_{m}^{+}(\omega): \omega \in M_{\delta, r}\right\}=\mu_{m}^{+}\left(\rho_{0}\right)=\mu_{m}\left(\rho_{0}\right)=\mathbf{L}_{m, \gamma}(r) .
$$

That is, the functional $\mu_{m}^{+}: M_{r, \delta} \rightarrow \mathbb{R}$ attains its minimum at $\rho_{0}$. 
Proof. Let us notice the following simple facts:

$$
\begin{aligned}
& \omega \in \mathcal{L}^{\gamma} \Longrightarrow \omega_{+} \geq \omega \text { and }\left\|\omega_{+}\right\|_{\gamma} \leq\|w\|_{\gamma}, \\
& \omega \in B_{\delta} \Longrightarrow \omega_{+} \in B_{\delta} .
\end{aligned}
$$

The latter fact is obtained from the elementary inequality $\left|\omega_{+}(t)-\rho_{0}(t)\right| \leq \mid \omega(t)-$ $\rho_{0}(t) \mid$ because $\rho_{0} \succ 0$.

If (5.12) is false, there exists $\omega \in M_{\delta, r}$ such that

$$
\mu_{m}^{+}(\omega)<\mu_{m}^{+}\left(\rho_{0}\right)=\mu_{m}\left(\rho_{0}\right)=\mathbf{L}_{m, \gamma}(r) .
$$

Now we apply the monotonicity result of Lemma 4.4 to obtain

$$
\mu_{m}\left(\omega_{+}\right)=\mu_{m}^{+}\left(\omega_{+}\right) \leq \mu_{m}^{+}(\omega)<\mathbf{L}_{m, \gamma}(r) .
$$

Denote $\hat{r}:=\left\|\omega_{+}\right\|_{\gamma}$. Then $0<\hat{r} \leq\|\omega\|_{\gamma}=r$ and $\omega_{+} \in E_{\hat{r}}^{\gamma}$. Thus

$$
\mathbf{L}_{m, \gamma}(\hat{r}) \leq \mu_{m}\left(\omega_{+}\right)<\mathbf{L}_{m, \gamma}(r),
$$

a contradiction to Lemma $[5.2$

The set $M_{\delta, r}$ is a differentiable manifold of $\left(\mathcal{L}^{\gamma},\|\cdot\|_{\gamma}\right)$ of codimension one without boundary. Since $\mu_{m}^{+}(\omega)$ defines a continuously differentiable functional on the open ball $B_{\delta}$, now the Lagrangian multiplier method [27, Ch. 43] can be applied to (5.12). More precisely, the condition $\omega \in S_{r}$ can be rewritten as

$$
V(\omega):=\int_{0}^{1}|\omega(t)|^{\gamma} d t-r^{\gamma}=0 .
$$

Notice that $V(\omega)$ is continuously differentiable in $\omega \in\left(\mathcal{L}^{\gamma},\|\cdot\|_{\gamma}\right)$ because $\gamma>1$. In fact one has

$$
\partial_{\omega} V(\omega)(t)=\gamma \phi_{\gamma}(\omega(t)) .
$$

Now (5.12) shows that the minimizer $\rho_{0}$ of $\mu_{m}^{+}(\omega), \omega \in M_{\delta, r}$, under condition (5.13), must satisfy

$$
-\mu_{m}\left(\rho_{0}\right) \cdot\left|E_{m}^{+}\left(t ; \rho_{0}\right)\right|^{p}=\left.\partial_{\omega} \mu_{m}^{+}(\omega)\right|_{\omega=\rho_{0}}(t)=c \cdot \gamma \phi_{\gamma}\left(\rho_{0}(t)\right)
$$

for some constant $c \neq 0$. See (4.8) and (5.14) for differentials. Since $\rho_{0} \succ 0$, we have $\phi_{\gamma}\left(\rho_{0}(t)\right)=\left(\rho_{0}(t)\right)^{\gamma-1}$. Now the equality is

$$
\left(\rho_{0}(t)\right)^{\gamma-1}=\tilde{c}\left|E_{m}^{+}\left(t ; \rho_{0}\right)\right|^{p},
$$

where $\tilde{c}=-\mu_{m}\left(\rho_{0}\right) /(c \cdot \gamma)$ is necessarily positive. Let us introduce

$$
y(t):=\tilde{c}^{1 / p} E_{m}^{+}\left(t ; \rho_{0}\right) .
$$

Then we have

$$
\rho_{0}(t)=|y(t)|^{p /(\gamma-1)} .
$$

Moreover, $y(t)$ is an eigenfunction associated with $\nu:=\mu_{m}\left(\rho_{0}\right)=\mathbf{L}_{m, \gamma}(r)>0$; i.e., $y(t)$ satisfies (1.8) and

$$
\left(\phi_{p}\left(y^{\prime}\right)\right)^{\prime}+\nu \rho_{0}(t) \phi_{p}(y)=0 .
$$

Substituting (5.16) into (5.17), we finally know that $y(t)$ is a solution of the following superlinear autonomous Lagrangian equation:

$$
\left(\phi_{p}\left(y^{\prime}\right)\right)^{\prime}+\nu \phi_{p \gamma^{*}}(y)=0
$$

with a parameter $\nu>0$. Equation (5.18) is called the critical equation for $\rho_{0}$, written in the form of some special eigenfunction $y(t)$. 
In order to find $\mathbf{L}_{m, \gamma}(r)$, one needs to find all possible solutions $y(t ; \nu)$ of (5.18) that satisfy the Dirichlet boundary condition and have precisely $m-1$ zeros in $t \in(0,1)$ because of (5.15). Then one can use (5.16) and the condition $\left\|\rho_{0}\right\|_{\gamma}=r$ to yield an equation for $\nu$, whose solution gives $\mathbf{L}_{m, \gamma}(r)$. Since (5.18) is integrable, all of these can be accomplished in a simple way. See the treatment in 31 for extremal problems of eigenvalues of Sturm-Liouville operators.

In the following we use another idea to solve (5.18). Let us notice that $\mathbf{L}_{m, \gamma}(r)$ and equation (5.18) are related with the following Sobolev constants:

$$
\mathbf{K}(\alpha, p):=\inf _{u \in W_{0}^{1, p}(0,1)} \frac{\left\|u^{\prime}\right\|_{p}^{p}}{\|u\|_{\alpha}^{p}}
$$

where $\alpha \in[1, \infty]$. These constants have been found explicitly by G. Talenti. See, for example, [28, Formula (4.1)]. For instance, $\mathbf{K}(p, p)=\pi_{p}^{p}$ and $\mathbf{K}(\infty, p)=2^{p}$. As a function of $\alpha, \mathbf{K}(\alpha, p)$ is continuous in $\alpha \in[1, \infty]$.

For the case $\alpha \in(1, \infty)$, the equation for the minimizer $u$, which can be assumed to satisfy $\|u\|_{\alpha}=1$, of (5.19) is just

$$
\left(\phi_{p}\left(u^{\prime}\right)\right)^{\prime}+\mu \phi_{\alpha}(u)=0,
$$

where $\mu=\mathbf{K}(\alpha, p)$ is the first eigenvalue of equation (5.20) subject to the Dirichlet boundary conditions. See equation (4.2) of [28. Thus (5.18) coincides with (5.20) with the choice $\alpha=p \gamma^{*}$. Applying the result in 28] to (5.20), we can give the final answer to $\mathbf{L}_{m, \gamma}(r)$ for the case $\gamma \in(1, \infty)$. That is,

$$
\mathbf{L}_{m, \gamma}(r) \equiv m^{p} \cdot \frac{\mathbf{K}\left(p \gamma^{*}, p\right)}{r}, \quad r \in(0, \infty) .
$$

Case $\gamma=1$. In this case, $\mathbf{L}_{m, 1}(r)$ cannot be attained at any $\rho \in E_{r}^{1}$. However, we will see that $\mathbf{L}_{m, 1}(r)$ can be realized by $\lim _{\gamma \downarrow 1} \mathbf{L}_{m, \gamma}(r)$.

By the monotonicity of $\mathbf{L}_{m, \gamma}(r)$ in $\gamma$ (Lemma 5.4), we have

$$
\mathbf{L}_{m, 1}(r) \leq \lim _{\gamma \downarrow 1} \mathbf{L}_{m, \gamma}(r) .
$$

On the other hand, any $\rho \in E_{r}^{1}$ can be approximated by elements in $E_{r}^{\gamma}, \gamma>1$, in the sense that there exists $\rho^{\gamma} \in E_{r}^{\gamma}, \gamma \in(1, \infty)$, such that $\lim _{\gamma \rightarrow 1}\left\|\rho^{\gamma}-\rho\right\|_{1}=0$. See 31. Lemma 2.1]. By the continuity of weighted eigenvalues and the monotonicity of $\mathbf{L}_{m, \gamma}(r)$ in $\gamma$, we have

$$
\mu_{m}(\rho)=\lim _{\gamma \downarrow 1} \mu_{m}\left(\rho^{\gamma}\right) \geq \lim _{\gamma \downarrow 1} \mathbf{L}_{m, \gamma}(r) .
$$

Thus

$$
\mathbf{L}_{m, 1}(r)=\inf _{\rho \in E_{r}^{1}} \mu_{m}(\rho) \geq \lim _{\gamma \downarrow 1} \mathbf{L}_{m, \gamma}(r) .
$$

This, together with (5.22), implies that

$$
\mathbf{L}_{m, 1}(r)=\lim _{\gamma \downarrow 1} \mathbf{L}_{m, \gamma}(r)=m^{p} \cdot \frac{\mathbf{K}(\infty, p)}{r}=\frac{(2 m)^{p}}{r} .
$$

Theorem 5.6. For any $\gamma \in[1, \infty], m \in \mathbb{N}$ and $r \in(0, \infty)$, the extremal value $\mathbf{L}_{m, \gamma}(r)$ is given by formula (5.21). 
We end the paper with some remarks.

(i) When $\gamma=1$, the extremal value $\mathbf{L}_{m, 1}(r)$ of (5.23) can also be deduced from [18, 25]. To this end, let us notice that the union

$$
\bigcup_{h \in[r, \infty)} E_{r, h}
$$

is dense in $E_{r}^{1}$ in the space $\left(\mathrm{E}^{1},\|\cdot\|_{1}\right)$. Using the continuity of eigenvalues, one has then

$$
\mathbf{L}_{m, 1}(r)=\lim _{h \rightarrow+\infty} \mathbf{L}_{m}(r, h) .
$$

Let $h \rightarrow+\infty$ in (5.3) and (5.4). One sees that the limit of $\mathbf{L}_{m}(r, h)$ in (5.24) is the same as that in (5.23).

(ii) Though (5.23) and (5.24) lead to the same conclusion for $\mathbf{L}_{m, 1}(r)$, the methods are quite different. Roughly speaking, (5.24) is proved by approximating the non-compact set $E_{r}^{1}$ by compact ones $E_{r, h}$. In this case, it is also very difficult to find the minimizers of (5.2). In (5.23), the set $E_{r}^{1}$ in the $L^{1}$ space is approximated by the sets $E_{r}^{\gamma}$ in the $L^{\gamma}$ spaces, $\gamma \in(1, \infty)$. By the results of this paper, the minimizers in $E_{r}^{\gamma}$ can be found via the critical equations. The present method is also used in [31] to find the extremal values of eigenvalues of linear operators. To the knowledge of the authors, although such an approach is quite natural, it has not been applied to extremal problems of eigenvalues before.

\section{REFERENCES}

1. S. Aizicovici, N. S. Papageorgiou and V. Staicu, Multiple nontrivial solutions for nonlinear periodic problems with the p-Laplacian. J. Differential Equations 243 (2007), 504-535. MR:2371798 (2008k:34166)

2. A. Anane, O. Chakrone and M. Monssa, Spectrum of one dimensional p-Laplacian with indefinite weight. Electr. J. Qualit. Th. Differential Equations, 2002 (2002), no. 17, 11 pp.

3. V. I. Arnold, Mathematical Methods of Classical Mechanics, Second edition, Graduate Texts Math., Vol. 60, Springer-Verlag, New York, 1989. MR997295 (90c:58046)

4. P. L. Binding and P. Drábek, Sturm-Liouville theory for the p-Laplacian. Stud. Sci. Math. Hungar. 40 (2003), 375-396. MR2037324 (2004j:34068)

5. P. L. Binding and B. P. Rynne, The spectrum of the periodic p-Laplacian. J. Differential Equations 235 (2007), 199-218. MR2309572 (2008m:35093)

6. P. Binding and B. Rynne, Variational and non-variational eigenvalues of the p-Laplacian. J. Differential Equations 244 (2008), 24-39. MR2373652 (2008k:34323)

7. H. W. Broer and M. Levi, Geometric aspects of stability theory for Hill's equations. Arch. Rat. Mech. Anal. 131 (1995), 225-240. MR1354696 (96h:58094)

8. M. Cuesta, Eigenvalue problems for the p-Laplacian with indefinite weights. Electr. J. Differential Equations, 2001 (2001), No. 33, 9 pp. MR1836801(2002b:35165)

9. Z. Denkowski, L. Gasinski and N. S. Papageorgiou, Positive solutions for nonlinear periodic problems with the scalar p-Laplacian. Set-Valued Anal. 16 (2008), 539-561. MR2465505

10. N. Dunford and J. T. Schwartz, Linear Operators, Part I, General Theory. Interscience, New York, 1958. MR0117523(22:8302)

11. W. Eberhard and Á. Elbert, On the eigenvalues of half-linear boundary value problems. Math. Nachr. 213 (2000), 57-76. MR1755246 (2001b:34035)

12. H. Feng and M. Zhang, Optimal estimates on rotation number of almost periodic systems. Z. Angew. Math. Phys. 57 (2006), 183-204. MR2214068 (2006k:37110)

13. J. K. Hale, Ordinary Differential Equations, John Willey, New York, 1969. MR0419901 $(54: 7918)$

14. P. Jebelean and N. S. Papageorgiou, Existence of solutions for a class of nonvariational quasilinear periodic problems. Set-Valued Anal. 16 (2008), 923-941. MR2466029 
15. R. Kajikiya, Y.-H. Lee and I. Sim, One-dimensional p-Laplacian with a strong singular indefinite weight, I, Eigenvalues. J. Differential Equations 244 (2008), 1985-2019. MR 2409516 (2009c:34022)

16. S. Karaa, Sharp estimates for the eigenvalues of some differential equations. SIAM J. Math. Anal. 29 (1998), 1279-1300. MR1628259 (99d:34162)

17. A. Katok and B. Hasselblatt, Introduction to the Modern Theory of Dynamical Systems, Cambridge Univ. Press, Cambridge, 1995. MR.1326374 (96c:58055)

18. M. G. Krein, On certain problems on the maximum and minimum of characteristic values and on the Lyapunov zones of stability. Amer. Math. Soc. Transl. Ser. 21 (1955), 163-187. MR0073776 (17:484e)

19. W. Li and K. Lu, Rotation numbers for random dynamical systems on the circle. Trans. Amer. Math. Soc. 360 (2008), 5509-5528. MR2415083

20. Y. Lou and E. Yanagida, Minimization of the principal eigenvalue for an elliptic boundary value problem with indefinite weight, and applications to population dynamics. Japan J. Indust. Appl. Math. 23 (2006), 275-292. MR.2281509(2008i:35044)

21. W. Magnus and S. Winkler, Hill's Equations, corrected reprint of 1966 edition, Dover, New York, 1979. MR559928 (80k:34001)

22. G. Meng and M. Zhang, Continuity in weak topology: First order linear systems of ODE. Acta Math. Sinica Engl. Ser. 26 (2010), 1287-1298. MR2657802

23. M. Möller and A. Zettl, Differentiable dependence of eigenvalues of operators in Banach spaces. J. Operator Theory 36 (1996), 335-355. MR1432122 (98d:47017)

24. J. Pöschel and E. Trubowitz, The Inverse Spectral Theory, Academic Press, New York, 1987. MR894477 (89b:34061)

25. P. Yan and M. Zhang, Best estimates of weighted eigenvalues of one-dimensional p-Laplacian. Northeast. Math. J. 19 (2003), 39-50. MR2011510 (2004j:34193)

26. C. Zanini, Rotation numbers, eigenvalues and the Poincaré-Birkhoff theorem. J. Math. Anal. Appl. 279 (2003), 290-307. MR1970507 (2004b:34115)

27. E. Zeidler, Nonlinear Functional Analysis and its Applications, III, Variational Methods and Optimization, Springer-Verlag, New York, 1985. MR768749 (90b:49005)

28. M. Zhang, Nonuniform nonresonance of semilinear differential equations. J. Differential Equations 166 (2000), 33-50. MR.1779254 (2001k:34043)

29. M. Zhang, The rotation number approach to eigenvalues of the one-dimensional p-Laplacian with periodic potentials. J. London Math. Soc. (2) 64 (2001), 125-143. MR.1840775 (2002e:35188)

30. M. Zhang, Continuity in weak topology: Higher order linear systems of ODE. Sci. China Ser. A 51 (2008), 1036-1058. MR2410982 (2009d:34012)

31. M. Zhang, Extremal values of smallest eigenvalues of Hill's operators with potentials in $L^{1}$ balls. J. Differential Equations 246 (2009), 4188-4220. MR.2517767 (2010d:34213)

Department of Mathematical Sciences, Tsinghua University, Beijing 100084, People's Republic of China

E-mail address: pyan@math.tsinghua.edu.cn

Department of Mathematical Sciences, Tsinghua University, Beijing 100084, People's Republic of China - and - Zhou Pei-Yuan Center for Applied Mathematics, Tsinghua University, Beijing 100084, People's Republic of China

E-mail address: mzhang@math.tsinghua.edu.cn 